\title{
EchoGéo
}

45 | 2018

Déclinaisons géographiques du changement social en Iran

\section{Représenter l'espace par la carte dans les manuels scolaires en Iran (1973-2012)}

\section{Alice Bombardier}

\section{OpenEdition}

\section{Journals}

Édition électronique

URL : https://journals.openedition.org/echogeo/15945

DOI : 10.4000/echogeo. 15945

ISSN : 1963-1197

Éditeur

Pôle de recherche pour l'organisation et la diffusion de l'information géographique (CNRS UMR 8586)

Référence électronique

Alice Bombardier, « Représenter l'espace par la carte dans les manuels scolaires en Iran

(1973-2012) », EchoGéo [En ligne], 45 | 2018, mis en ligne le 05 novembre 2018, consulté le 10 août 2021. URL : http://journals.openedition.org/echogeo/15945; DOI : https://doi.org/10.4000/echogeo. 15945

Ce document a été généré automatiquement le 10 août 2021.

EchoGéo est mis à disposition selon les termes de la licence Creative Commons Attribution - Pas d'Utilisation Commerciale - Pas de Modification 4.0 International (CC BY-NC-ND) 


\title{
Représenter l'espace par la carte dans les manuels scolaires en Iran (1973-2012)
}

\author{
Alice Bombardier
}

1 Au début des années 1990, faute de recensement officiel, diverses enquêtes réalisées par l'État iranien estimaient que les Persans, établis surtout sur le plateau central du pays, représentaient seulement $46 \%$ de la population. Les minorités de la périphérie étaient donc dans leur ensemble quantitativement plus nombreuses, avec principalement les turcophones $21 \%$, les Kurdes $10 \%$, les Lors $9 \%$, les populations caspiennes $7 \%$, les Arabes 3,5\% et les Baloutches $3 \%$ (Hourcade, 2002, p. 32). Dans cet article, nous interrogerons dès lors le rôle joué par l'enseignement de la géographie en Iran, à la fois dans la définition du territoire iranien et dans la transmission d'un sentiment national fort. Comment la géographie scolaire contribue-t-elle à «faire territoire » et à édifier une nation sur des bases si largement pluriethniques ?

2 Pour ce faire, nous nous concentrerons sur le prisme de la représentation cartographique. Les savoirs cartographiés sont représentés dans les manuels scolaires en Iran via des procédés de visualisation qui cristallisent du sens - de la sémantique consciente à l'imprégnation inconsciente - à différents niveaux : ils semblent tout à la fois faire office de traits identitaires, signes de reconnaissance, valeurs d'échange, instruments de pouvoir et liens communautaires. À l'appui de huit manuels scolaires édités entre 1973 et 2012 à Téhéran ${ }^{1}$ - quatre manuels de persan du primaire (1973-1979) et quatre manuels de géographie du secondaire (1980-2012) -, seront mis en évidence des objets géographiques, des formes graphiques et plus généralement des scénarios cartographiques ${ }^{2}$ qui, inscrits dans la spécificité de l'Iran, sont investis en milieu scolaire d'une valeur paradigmatique, véhiculant un discours de référence et des représentations.

3 La grille d'analyse a consisté tout d'abord à référencer les «habits de la carte» (Brunet, 1987, p.62), incluant les modalités de projection, centrage, orientation, échelle, contours, titre ou légende ; puis à cerner l'espace géographique concerné, la 
nature des découpages opérés, mais aussi le lien de proximité ou non attribué à cet espace; à s'intéresser à la sémiologie cartographique, notamment à la nature, à la fréquence ou à l'absence de certains signes graphiques; enfin, à l'association avec le texte, c'est-à-dire au champ icono-textuel.

4 Cette lecture de la carte dans la géographie scolaire iranienne ne peut être dissociée d'une réflexion sur les développements de la science géographique hors d'Europe. Les études géographiques à l'échelle mondiale ont longtemps été coloniales, imprégnées du regard occidental. Aujourd'hui, les programmes de recherche tendent à reconnaître la trajectoire en propre des géographies extra-occidentales. Ce cheminement spécifique a érigé la géographie à une place de choix dans le système éducatif iranien et mérite de ce fait qu'on s'y attarde. Après un retour sur ce contexte scolaire, nous montrerons quelles représentations du territoire national et du monde sont véhiculées par la carte dans les manuels étudiés.

\section{La géographie et la carte dans le système scolaire iranien}

\section{La géographie en révolution?}

Le système scolaire iranien est tributaire du modèle français, dont il s'est inspiré dans les années 1920 lors de la création du premier système éducatif public. Auparavant, la géographie - malgré les développements de cette science à l'époque médiévale (Tixier du Mesnil, 2010) - n'apparaissait pas dans les curricula des établissements d'enseignement traditionnels (maktab et madreseh) ${ }^{3}$. Les premières occurrences de l'enseignement de la géographie en tant que discipline scolaire sont intervenues en Iran dans le cadre des écoles missionnaires, dont la première - américaine et protestante - a été implantée à Orumiyeh en 1834. Des écoles lazaristes françaises ont été ouvertes quelques années plus tard. Chaque mission suivait l'exemple et le modèle éducatif de son pays respectif (Paivandi, 2008; Hellot-Bellier, 2012). La géographie a ensuite fait partie des curricula publics conçus sous Reza Shah Pahlavi au cours des années 1920 (Birashk, 1998a, p. 203). Aujourd'hui, elle est abordée dès la première année du primaire dans les manuels de langue persane, même si son enseignement ne commence véritablement qu'en $3^{\mathrm{e}}$ année du primaire.

6 La place de la géographie dans les curricula iraniens a peu varié depuis l'instauration du système éducatif public : cette discipline garde un statut important, indépendant de l'histoire et parfois se substituant à elle à la fin du secondaire (l'équivalent du lycée français) ${ }^{4}$. Mais le contenu de son enseignement a connu des changements significatifs. La révolution culturelle qui bouleverse le pays dans les années 1980 a entraîné des modifications nombreuses aussi bien des programmes que des manuels scolaires (Anquetil, 1978 ; Bombardier, 2013). Le lien entre l'éducation et la religion islamique a été clairement édicté dans le premier plan éducatif (Tarh-e kolliyat-e nezam-e amuzesh o parvaresh-e djomhuri-e eslami-e Iran) publié par le nouveau Ministère de l'Enseignement et de l'Éducation. L'Iran a connu alors « une expérience de délaïcisation " (Paivandi, 2014). Après plusieurs décennies de laïcisation du savoir scolaire sous la dynastie Pahlavi (1925-1979), l'indépendance du système éducatif vis-à-vis de l'institution religieuse a été remise en question. Le système éducatif est aujourd'hui non seulement basé sur les enseignements religieux, sur le rejet de toute forme d'athéisme ou de 
polythéisme, mais aussi orienté vers la restauration de la culture et de la civilisation islamique contre la « culture coloniale et occidentale » (Mehran, 1998, p. 230).

7 Le nouveau régime a bouleversé les paramètres idéologiques et les curricula des enseignements mais n'a que peu réformé les contenus scientifiques et les principes pédagogiques. En 1997, A. Birashk observe que seulement $10 \%$ des manuels utilisés ont été écrits après la Révolution, $90 \%$ étant des versions révisées des livres scolaires de l'époque impériale, expurgés de toute allusion à l'ancien régime, de références culturelles étrangères, de photos de femmes non voilées (Birashk, 1998b, p. 215). Aujourd'hui, certains auteurs déplorent l'aspect sclérosé des manuels scolaires en circulation dans le pays (Ashraf, 1998, p. 196).

\section{La carte dans le système d'images}

8 La carte constitue l'illustration principale de ces manuels scolaires. L'omniprésence du document cartographique est flagrante dans les quatre manuels de notre corpus dédiés à la géographie. Dans celui de 1980 voué à la géographie de l'Iran, $91 \%$ des doublespages (entendues ici comme deux pages qui se font face) comptent au moins une carte. Cette proportion est la même pour les manuels de $2^{\mathrm{e}}$ année du secondaire en 1981 et 2012 présentant le monde, tandis que celui de $3^{\mathrm{e}}$ année en 2012 , consacré à des principes généraux de climatologie, géologie et astronomie, met l'accent sur l'explication des phénomènes plutôt que sur leur localisation. Le nombre de cartes y est réduit même si plus de la moitié (62 \%) des doubles-pages en compte encore. La carte est souvent identifiée à la discipline géographique (Fontanabona et alii, 2002, p. 168). Elle joue un rôle de "marqueur identitaire $»^{5}$ et contribue à la légitimation de la géographie au sein de l'éventail des disciplines scolaires. Il demeure que l'approche géographique en milieu scolaire dans l'Iran post-révolutionnaire s'avère fondamentalement scopophile, désireuse de donner à voir l'espace, mais privilégie un regard indirect sur le monde en recourant essentiellement à la carte.

9 En milieu scolaire, la carte comme représentation (l'acte de faire une carte) doit être distinguée de la carte comme figuration (la carte utilisée comme image) (Chevalier, 1993). Seul le manuel de $3^{e}$ année du secondaire en 2012 commence par une activité de travaux pratiques: la confection d'un globe terrestre sur un ballon, ce qui permet d'étudier les déformations générées par la projection. Les autres manuels scolaires étudiés ne donnent pas à dessiner de carte mais intègrent celle-ci comme image. La récurrence de ce support visuel et sa fonction surtout descriptive ${ }^{6}$ aboutissent à un processus d'autoréférence cartographique: échelle et orientation, légende et titre apparaissent très peu. Dans le manuel de 1980, un même fond de carte est complété de façon séquentielle sur l'ensemble du manuel au point que les outils d'identification de l'espace (toponymes) et de la carte (titre, échelle, orientation, légende) ne sont plus indiqués. L'absence récurrente de ces attributs cartographiques laisse supposer une autre finalité de la carte : discursive. La carte sert-elle davantage à appuyer un discours qu'à représenter l'espace?

10 En français, «carte » vient du latin charta, tiré du grec khartès, qui signifie « feuille de papyrus » (Brunet, 1987, p. 8). L'accent est mis sur le medium. En persan, le mot «carte» a un sens plus proche de celui d'«image » et d'«illustration»: il s'écrit naqsheh, terme qui dérive de naqsh " dessin ", " peinture ». Cette conception singulière de la carte dans le contexte persan contribue à expliquer sa fonction descriptive mais 
aussi l'emploi récurrent du pictogramme comme principal signe graphique. À l'opposé des chorèmes abstraits de R. Brunet et de la recherche de scientificité de la cartographie française, les nombreuses cartes en symboles de ces manuels traduisent la priorité donnée en Iran à l'iconicité du document cartographique. La dimension subjective est forte : ces cartes associent d'emblée images mentales et sentiments aux lieux. Elles concourent à la fabrique d'un territoire-icône ${ }^{7}$. Voyons dès lors les territorialités condensées dont regorge cette cartographie scolaire pour représenter l'espace national.

\section{La carte dans l'iconographie du sentiment national}

\section{L'empreinte du tracé des frontières}

L'Iran a perdu du territoire à plusieurs reprises au cours du XIX ${ }^{e}$ siècle du fait des conflits l'opposant aux puissances occidentales. Dans les années 1970, la frontière entre l'Iran et l'Irak est encore discutée. À l'époque des accords d'Alger (1975-1976), qui tentent de mettre fin à ces disputes territoriales, un fond de carte est introduit dans un manuel de persan du primaire pour mémoriser la forme du tracé des frontières nationales. Ce même tracé simplifié est reproduit en 1980 un grand nombre de fois dans le manuel de géographie du secondaire (illustrations 1 et 2). Ces cartes témoignent d'une inter-iconicité existant entre manuels d'époques et de niveaux différents, ici dans un contexte pré- et post-révolutionnaire.

Illustration 1 - Leçon « Patrie »

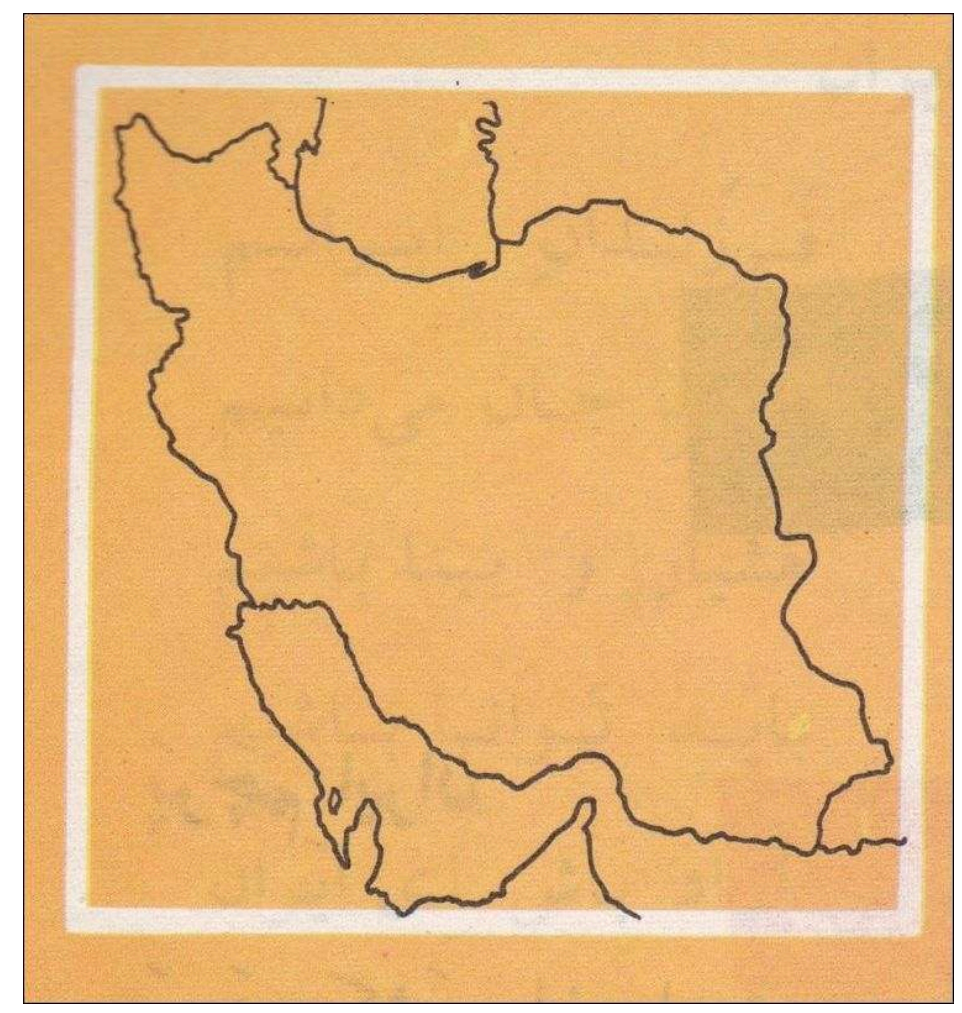

Source : Manuel de persan, Primaire, 1 ère année, 1976, p. 89. 
Illustration 2 - Leçon « Caractéristiques de la faune et de la flore en Iran »

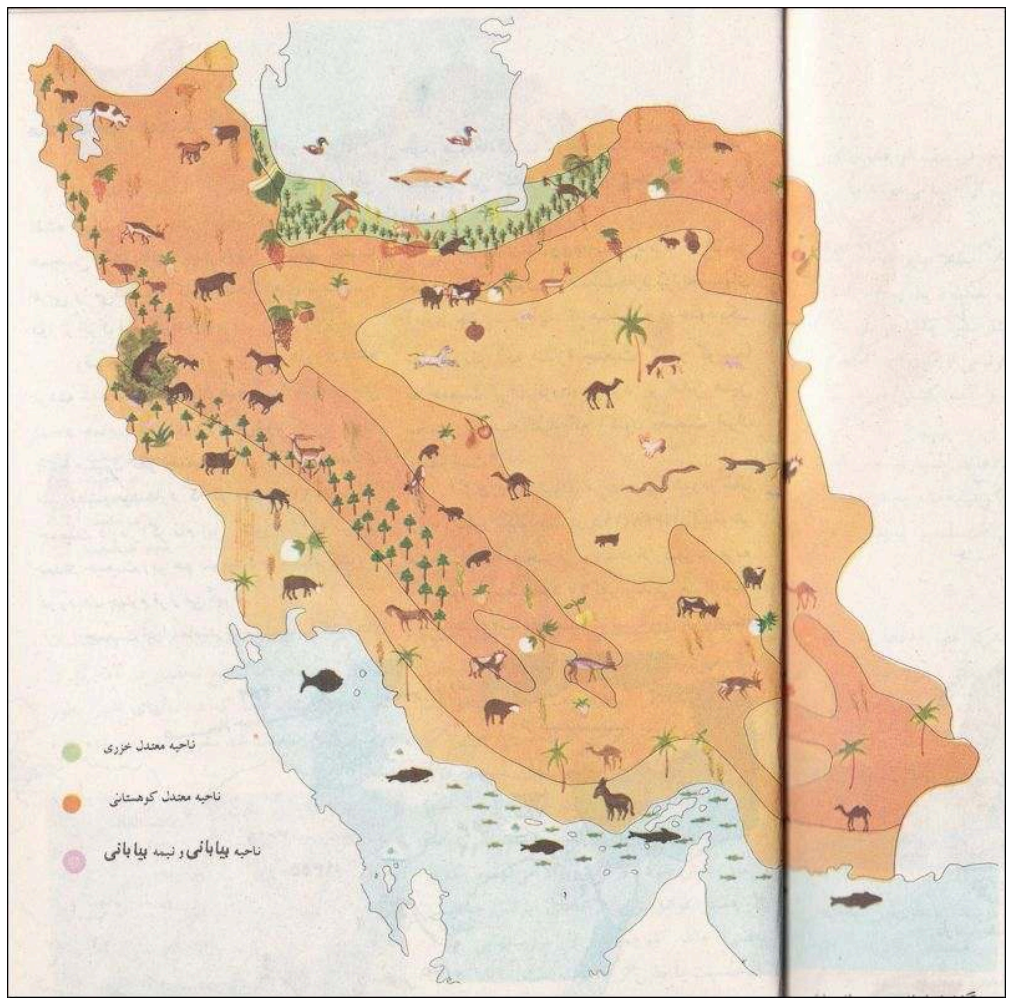

Source : Manuel de géographie, Secondaire, 7 ère année, 1980, p. 148-149.

12 Les outils d'identification de l'espace ne sont pas indiqués, l'autoréférence cartographique est manifeste: ces cartes ne s'avèrent intelligibles que grâce à la mémorisation préalable $d u$ tracé des frontières $d u$ pays $^{8}$. Ce phénomène peut s'apparenter à une « empreinte » au sens psychologique. Définie par K. Lorenz dans les années 1930, l'empreinte ou imprégnation dénote le lien psychologique instauré entre une forme spécifique qui sert de déclencheur extérieur et un état d'esprit ou comportement instinctif.

13 L'importance donnée à la visualisation du territoire national, à la mémorisation du tracé des frontières et de ses proportions fait d'ailleurs l'objet d'activités spécifiques. La leçon introductive du manuel de 1980 est consacrée à la « forme et aux dimensions de l'Iran " (Shekl-e Iran va andazeh-ye an). Cette leçon (illustration 3) est l'occasion d'une activité à basse intensité intellectuelle où il est demandé à l'élève de décrire les formes (parfaites) du losange ${ }^{9}$ qui est utilisé pour symboliser le territoire national. 
Illustration 3 - Leçon « Forme de l'Iran et ses dimensions »

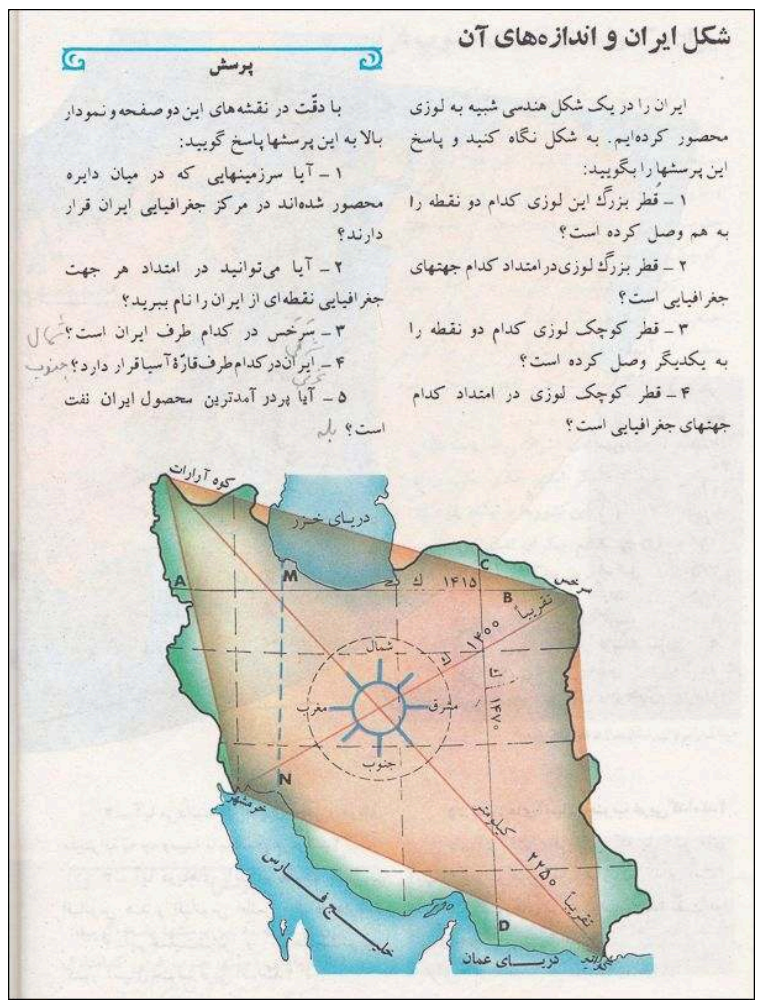

Nous enserrons l'Iran dans une forme géométrique ressemblant à un losange. Observez cette forme et répondez aux questions:

1. Quels sont les deux points joints par la grande diagonale (qotr) de ce losange?

2. Dans le prolongement de quelles directions géographiques (djahatha-ye djeografia'i) est la grande diagonale de ce losange?

3. Quels sont les deux points joints par la petite diagonale de ce losange?

4. Dans le prolongement de quelles directions géographiques est la petite diagonale de ce losange?

Source : Manuel de géographie, Secondaire, 7 ère année, 1980, p. 12.

Aux extrémités de ce losange, bornant le territoire, sont indiquées trois villes Khorramshahr au Sud-Ouest, Guvantar au Sud-Est et Sarakhs au Nord-Est - et un élément de relief - la montagne Ararat au nord-ouest. La distance réelle équivalente aux diagonales du losange est spécifiée : $1400 \mathrm{~km}$ pour la petite diagonale et $2250 \mathrm{~km}$ pour la grande. L'accent est mis sur l'orientation de ces diagonales avec l'indication des points cardinaux en leur centre. Il s'avère que ce « centre » correspond à la province du Fars, espace d'origine de l'ethnie persane qui y est majoritaire : le centre géographique correspond aussi à un centre identitaire. Le sentiment national prend donc appui sur la représentation cartographique, qui peut être répétée selon les mêmes contours tout au long de la scolarité. Le recours à une forme géométrique et au tracé de traits mathématiques bien proportionnés permet en outre de véhiculer l'idée d'une perfection de la taille et de la forme du territoire. Cette démarche iconique est similaire en France, où le territoire national est symbolisé par un hexagone. Dans les deux cas, cette démarche permet de justifier le tracé des frontières et de donner l'impression d'une centralité naturelle du territoire. 


\section{Amour de la patrie et/ou attachement régional ?}

Cette mémorisation du tracé des frontières contribue à l'instillation d'un fort sentiment d'appartenance au cadre national. Dans le manuel de 1976, un texte aux accents nationalistes intitulé « Patrie » (Mihan), auquel est apposé un fond de carte du territoire national, témoigne de l'importance accordée à cette échelle d'appartenance. Après la Révolution, un texte similaire est publié avec une carte des principales villes du pays (illustrations 4 et 5).

Illustration 4 - Leçon « Patrie » en 1976

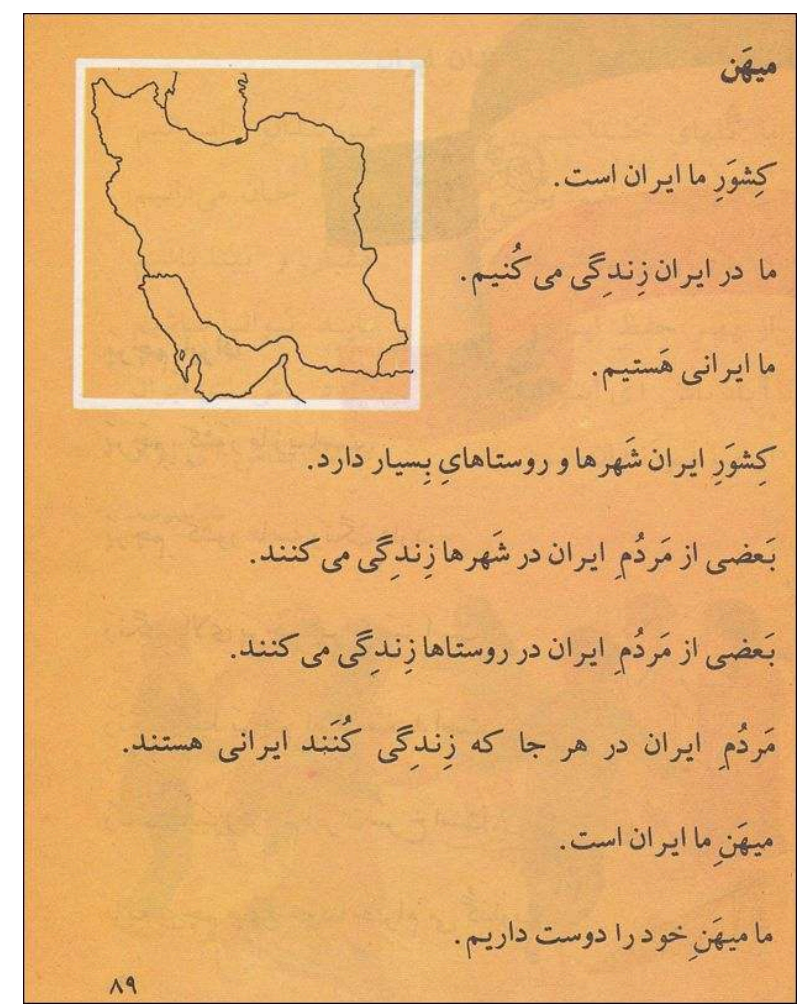

Patrie (Mihan)

Notre pays est l'Iran.

Nous vivons en Iran.

Nous sommes Iraniens.

Le pays Iran a beaucoup de villes et de villages.

Certains habitants de l'Iran vivent en ville.

Certains habitants de l'Iran vivent dans des villages.

Les habitants de l'Iran peu importe là où ils habitent sont Iraniens.

Notre patrie est l'Iran.

Nous aimons notre patrie.

Source : Manuel de persan, Primaire, 1 ère année, 1976, p. 89. 


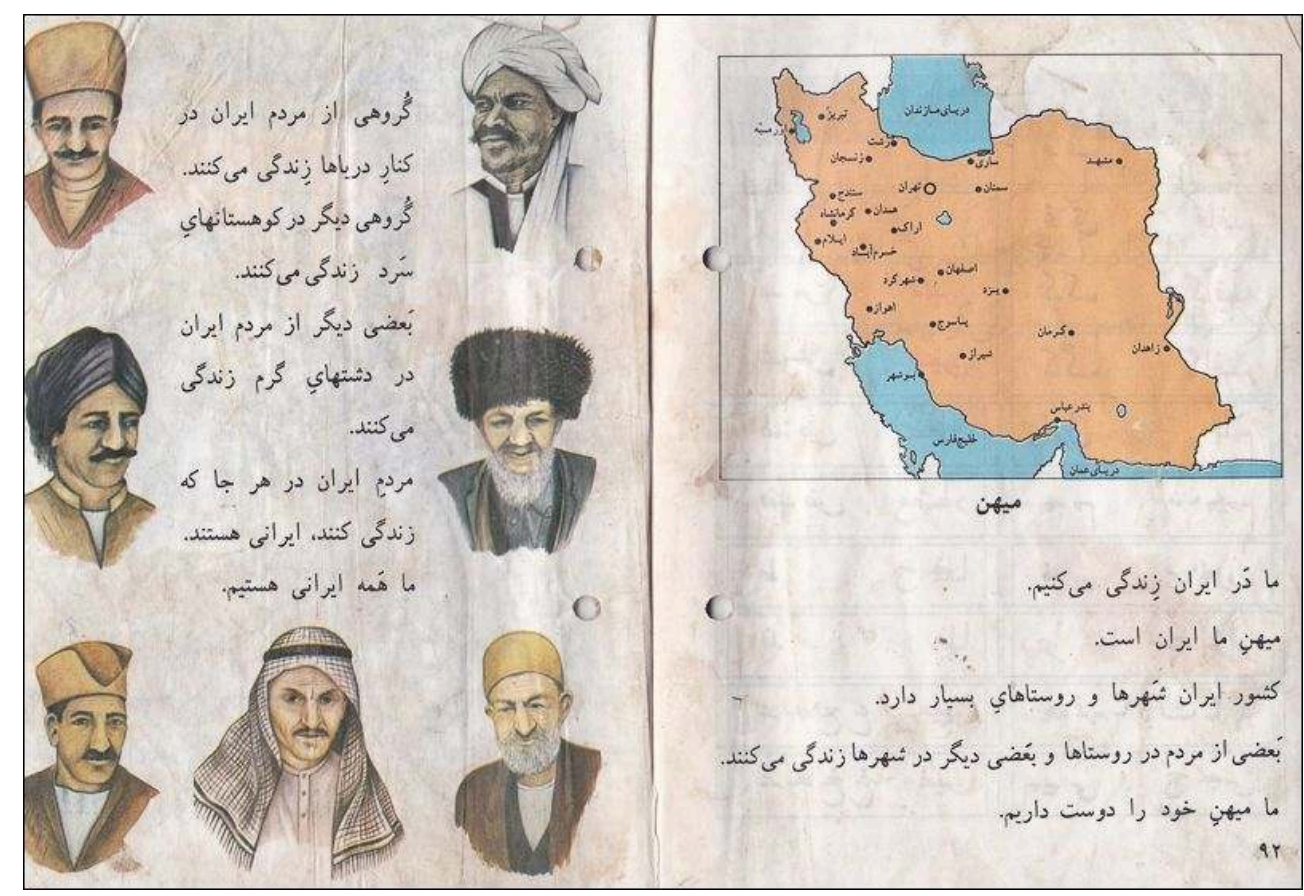

Patrie (Mihan)

Nous vivons en Iran.

Notre patrie est l'Iran.

L'Iran a beaucoup de villes et de villages.

Certains vivent dans les villages et d'autres dans les villes.

Nous aimons notre patrie.

Un groupe de la population de l'Iran vit à côté de la mer.

Un autre groupe vit dans des montagnes froides.

D'autres habitants de l'Iran vivent dans des plaines chaudes.

Les habitants de l'Iran peu importe là où ils vivent sont Iraniens.

Nous sommes tous Iraniens.

Source : Manuel de persan, Primaire, 1 ère année, 1979, p. 92-93.

Malgré un fort attachement exprimé envers la patrie dans son entier, le manuel postrévolutionnaire introduit des distinctions ethniques plus différenciées. Aux phrases " Nous vivons en Iran ", " Notre patrie est l'Iran » est ajouté un long passage sur la diversité des peuples iraniens, portraits folkloriques à l'appui. Leur implantation géographique est présentée selon des repères simples (mer, montagne, plaine) définissant de grandes régions naturelles. Selon P. Vidal de la Blache et L. Gallois, la région naturelle est une portion de l'espace terrestre dont la spécificité tient en effet à l'étroite combinaison de ses caractéristiques physiques, historiques et des activités des populations qui l'occupent ${ }^{10}$. Le texte précise toutefois que ces peuples, «tous Iraniens », font partie du cadre national. L'objectif essentiel de cette échelle d'appartenance régionale demeure d'ancrer en arrière-fond une identité nationale unificatrice.

La monarchie Pahlavi a lutté contre les particularismes ethniques et sédentarisé de force certaines tribus. La nécessité de connaître les spécificités de sa région est a contrario introduite par la République islamique. Selon N. Yavari-d'Hellencourt, qui a étudié le traitement particulier réservé à l'ethnie dans les manuels scolaires des années 1980, la République islamique a trouvé dans la valorisation de l'ethnicité un moyen de lutter contre l'« intrusion culturelle occidentale » et a favorisé ces modes de 
vie traditionnels qui s'avèrent être proches de la vision idéale d'une culture traditionnelle authentique. Ce sont les caractéristiques communes - partage de l'espace et/ou origine ethno-linguistique semblable - qui sont mises en avant pour intégrer ces minorités ethnico-religieuses (Yavari-d'Hellencourt, 1988, p. 260). Les deux cartes cidessous illustrent cette volonté du nouveau régime de renforcer la cohésion nationale autour de la jeune République islamique. La carte de l'illustration 7 montre un arbre dont les racines plongent dans la terre d'Iran tandis que les branches montent vers le ciel et portent l'emblème de la République islamique signifiant "Allah». Le tronc représente la Constitution (Qanun-e esasi est inscrit dessus). Cette illustration qui utilise la carte comme fondement et signe abstrait aisément reconnaissable rappelle que le discours scolaire en faveur de l'unité des ethnies est basé sur la Constitution de la République islamique, dont l'article 15 reconnaît la légalité de l'usage de toutes les langues et dialectes pratiqués dans le pays. Cependant, l'enseignement des langues régionales reste interdit dans les écoles publiques (Hourcade, 2002, p. 34).

Illustration 6 - Diversité ethnique, unicité de la nation

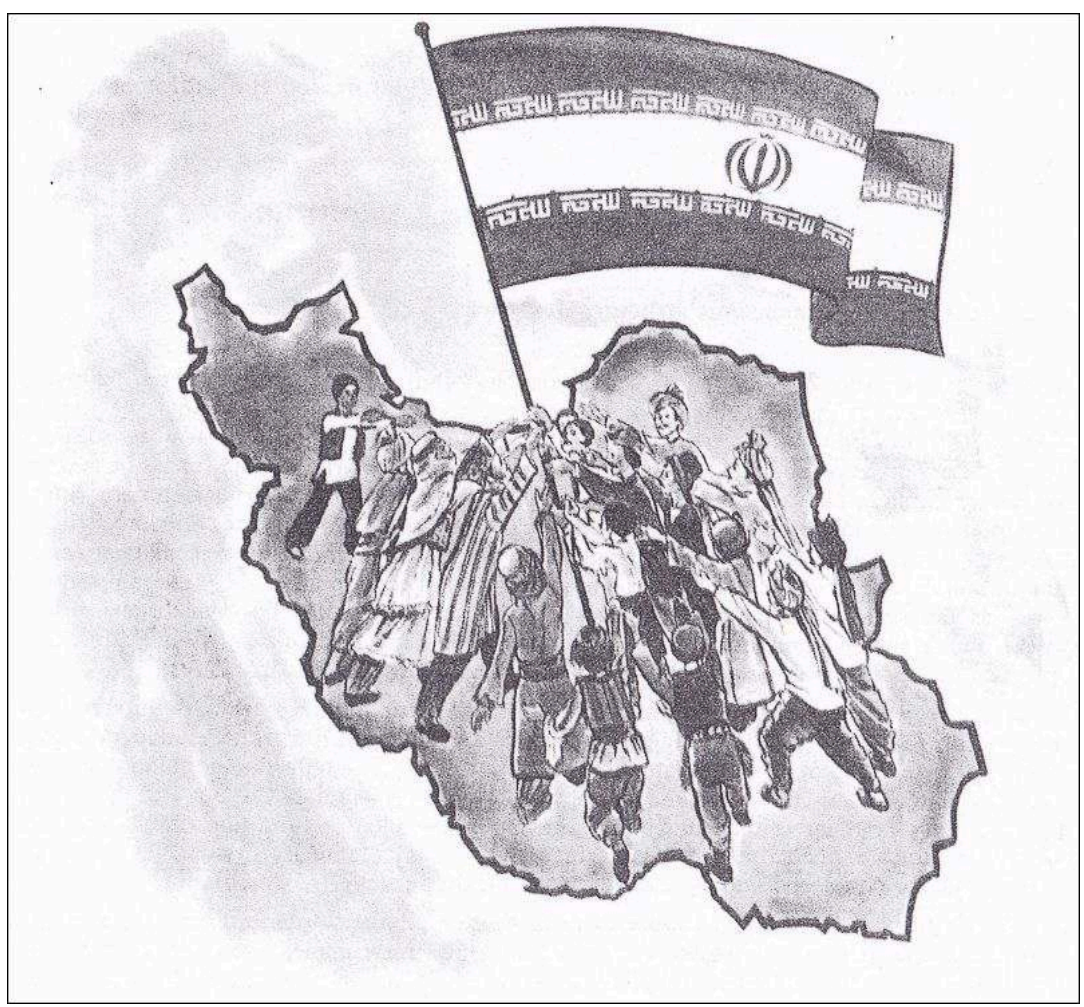

Source : Manuel de sciences sociales, $4^{e}$ année du Primaire, 1984-85, p. 170. In Yavari d'Hellencourt, 1988, p. 253 
Illustration 7 - Le peuple et la Constitution

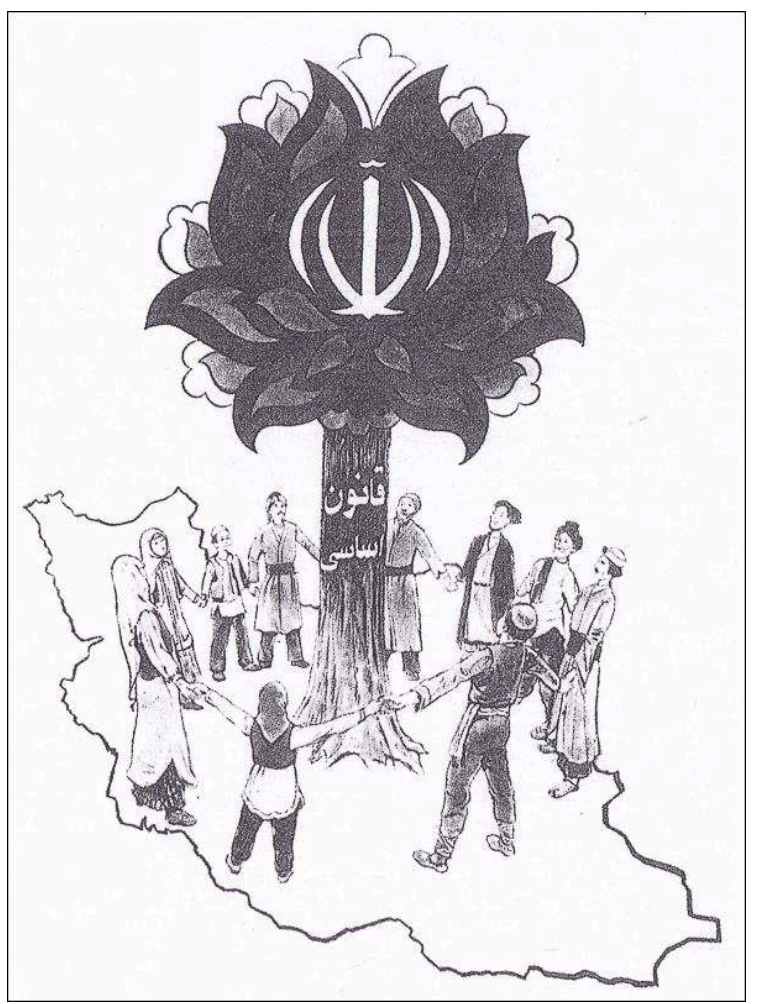

Source : Manuel de sciences sociales, 5e année du Primaire, 1984-85, p. 234. In Yavari d'Hellencourt, 1988, p. 257.

La présence de cette échelle d'appartenance régionale dans les manuels scolaires postrévolutionnaires s'inscrit donc dans une revalorisation globale de l'ethnicité et de l'identité régionale. Si dans les années 1980 la région naturelle constitue un cadre privilégié, la région administrative est également un découpage pratiqué. Des manuels géographiques par région administrative ont été édités au milieu des années $1980^{11}$. Mais cet attachement régional à géométrie variable reste clairement affilié à une volonté de renforcement de l'identité nationale ${ }^{12}$.

\section{Un pays sans capitale?}

Sous le régime impérial, Téhéran est lié à la politique de prestige et à la recherche de légitimation de la dynastie Pahlavi. Le manuel de 1976 consacre une leçon entière à la « capitale de l'Iran » (Paitakht-e Iran), présentée comme un fief du pouvoir en place : 
Illustration 8 - Leçon « La capitale de l'Iran » en 1976

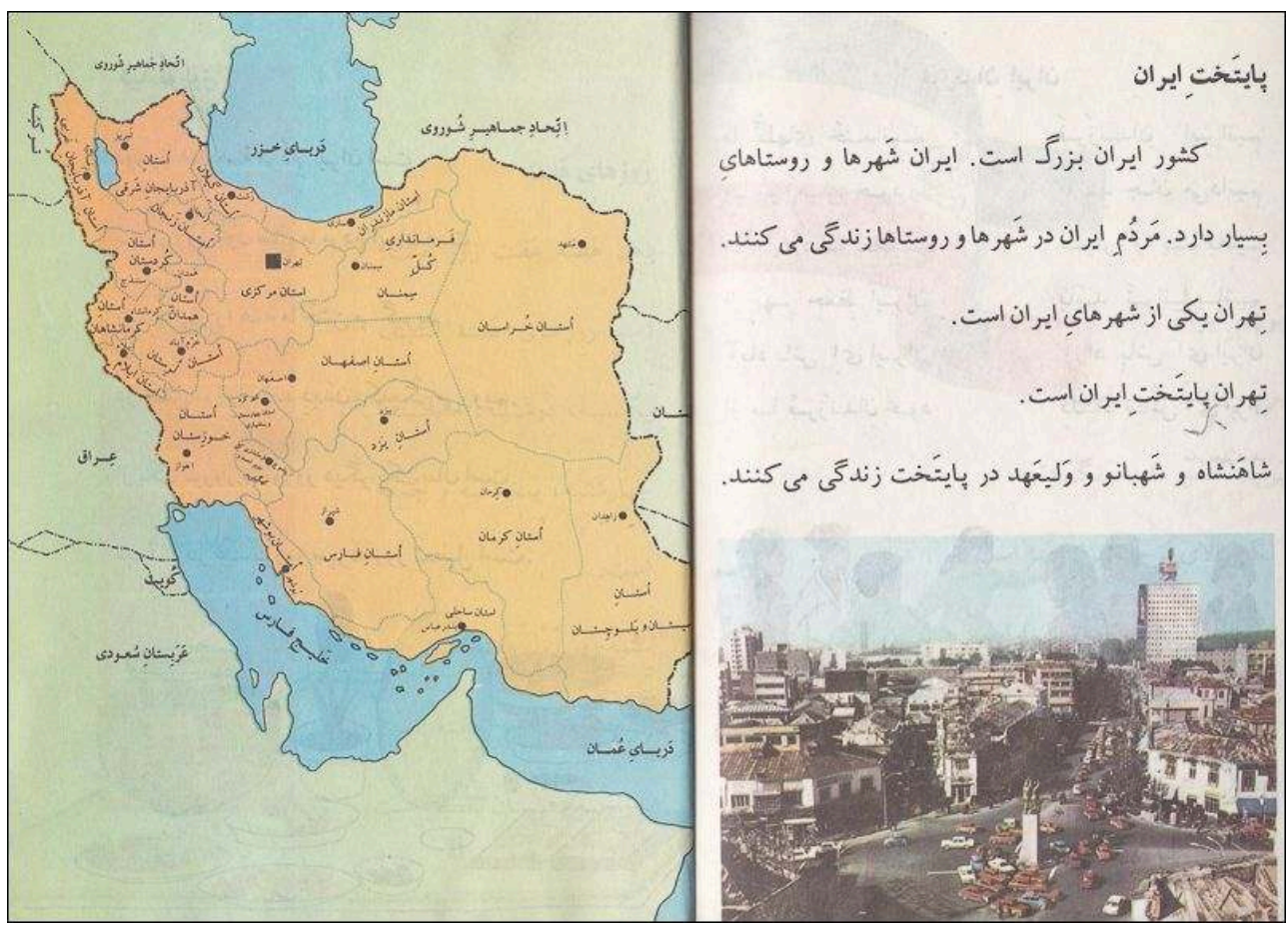

La capitale de l'IranLe pays Iran est grand. L'Iran a beaucoup de villes et de villages. La population de I'Iran vit dans des villes ou des villages. Téhéran est une des villes d'Iran. Téhéran est la capitale de I'Iran. Le Roi des Rois, la Reine et le prince héritier vivent dans la capitale.

Source : Manuel de persan, Primaire, 1 ère année, 1976, p. 92-93

$$
\begin{aligned}
& \text { niveau administratif en tant que capitale, à un niveau politique en tant que lieu de vie } \\
& \text { de la dynastie Pahlavi entendue selon une acception large, incluant déjà le prince } \\
& \text { héritier. }
\end{aligned}
$$
de la capitale : il s'agit sans doute de l'avenue Pahlavi ou de sa perpendiculaire, l'avenue Reza Shah. Ces avenues aujourd'hui rebaptisées Vali-e Asr et Enqelab sont les deux principales artères de Téhéran, aménagées sous Reza Shah Pahlavi. L'avenue Vali-e Asr s'étendant sur $18 \mathrm{~km}$ est considérée comme une des plus longues avenues du MoyenOrient. La longueur de l'avenue est mise en valeur par le point de vue aérien qui permet de la voir filer jusqu'à la ligne d'horizon. Au premier plan, un carrefour est le lieu d'un trafic de voitures sans doute important pour l'époque. Au deuxième plan, après les maisons traditionnelles à deux étages, apparaissent les premiers buildings avec réclames publicitaires. La population n'est pas représentée. C'est l'urbanisation de la ville et ses flux automobiles, c'est-à-dire l'aspect occidentalisé de la ville et son dynamisme, qui sont mis en avant. La page attenante est consacrée à une carte des régions administratives de l'Iran, où est indiqué pour chacune le chef-lieu (illustration 8). Pour la région de Téhéran, «région centrale» (Ostan-e markazi), la sémiologie graphique distingue Téhéran (un gros carré) des autres villes provinciales (un petit rond). Cette carte est l'occasion de hiérarchiser le maillage urbain du pays et de montrer que Téhéran est à sa tête.

En 1980, Téhéran ne fait plus l'objet de développement particulier. La capitale n'apparaît qu'à 11 reprises sur les cartes du manuel alors que celui-ci compte 88 cartes 
consacrées à l'Iran. Elle est bannie de la définition du cadre national, au point que le chapitre sur la vie urbaine portant sur la région montagneuse de Téhéran mentionne Tajrish comme toponyme et non Téhéran (illustration 9). Tajrish est le nom d'une ancienne ville de faubourg de Téhéran devenue aujourd'hui un quartier situé au nord de la capitale tentaculaire ${ }^{13}$. En outre, la carte des provinces administratives de ce manuel ne fait plus apparaître de hiérarchie entre Téhéran et les chefs-lieux de province (illustration 10).

Illustration 9 - Leçon « La vie urbaine » en 1980

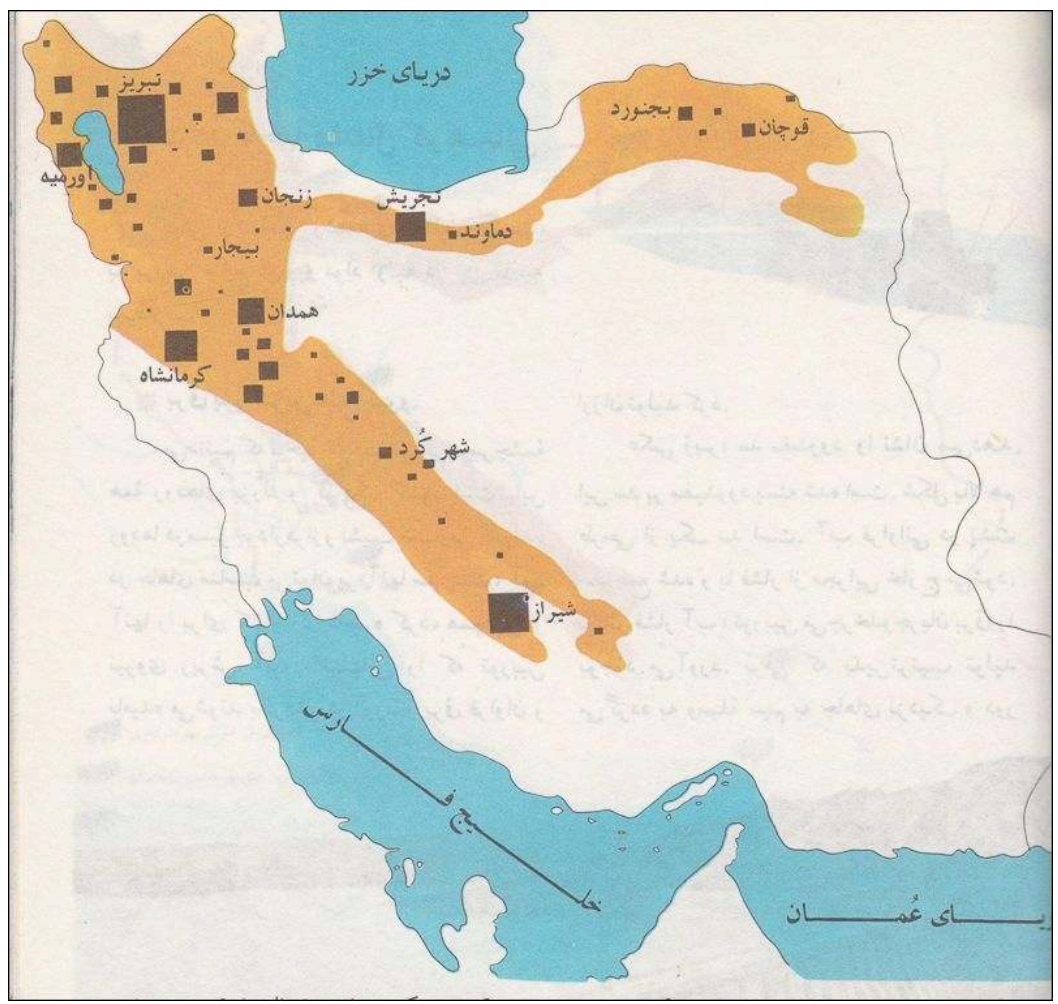

Source : Manuel de géographie, Secondaire, 1 ère année, 1980, p. 91. 
Illustration 10 - Leçon « La région dans laquelle nous vivons » en 1980

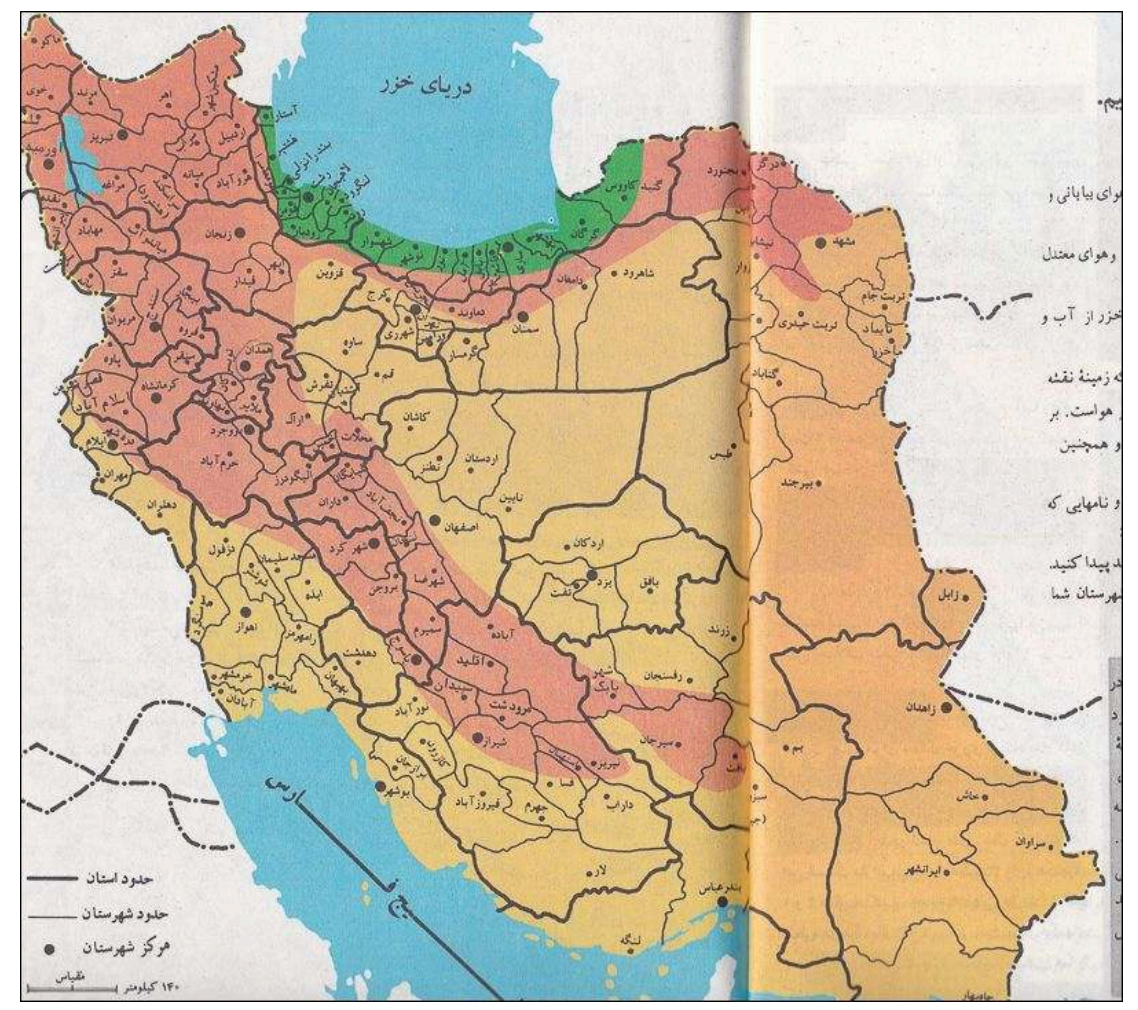

Source : Manuel de géographie, Secondaire, 7 ère année, 1980, p. 18-19.

Durant la Révolution culturelle, la capitale iranienne a subi une éviction symbolique. Cet escamotage cartographique recoupe la dénonciation des dérives de la vie urbaine que pratique la République islamique. Selon sa nouvelle rhétorique, les villes sont le théâtre d'une modernisation culturellement dévastatrice. En 1998, les manuels scolaires insistent sur ce malaise urbain et affichent une nette préférence pour la vie rurale : $70 \%$ des métiers évoqués dans les livres du primaire de 1996 correspondent aux secteurs de l'agriculture, de l'artisanat et du bâtiment ${ }^{14}$. Mais qu'en est-il, hors du seul cadre urbain et national, de la vision du monde qu'ils véhiculent?

\section{La carte dans la représentation du monde}

\section{Des projections non-occidentales}

Aucune des cartes du monde ( 5 seulement) que comptent les manuels étudiés ne reprend une projection de Mercator, d'abord répandue en Europe puis dans le reste du monde. Le manuel de 1980 s'ouvre sur un planisphère écorcé comme une orange selon la projection de Goode avec un centrage sur l'Afrique (illustration 11). Cette projection pseudo-cylindrique à aires égales, très répandue dans les années 1960, a été créée en 1923 comme alternative à la projection de Mercator. La vertu pédagogique de cette projection est de rendre compte de la distribution spatiale des continents. Dans un contexte de tension extrême avec les États-Unis (les otages américains sont détenus à Téhéran de novembre 1979 à janvier 1981), ce planisphère éclaté permet aussi de dissocier le continent eurasiatique du continent américain. 
Illustration 11 - Planisphère selon la projection de Goode en 1980. Leçon « L'Iran dans le monde »

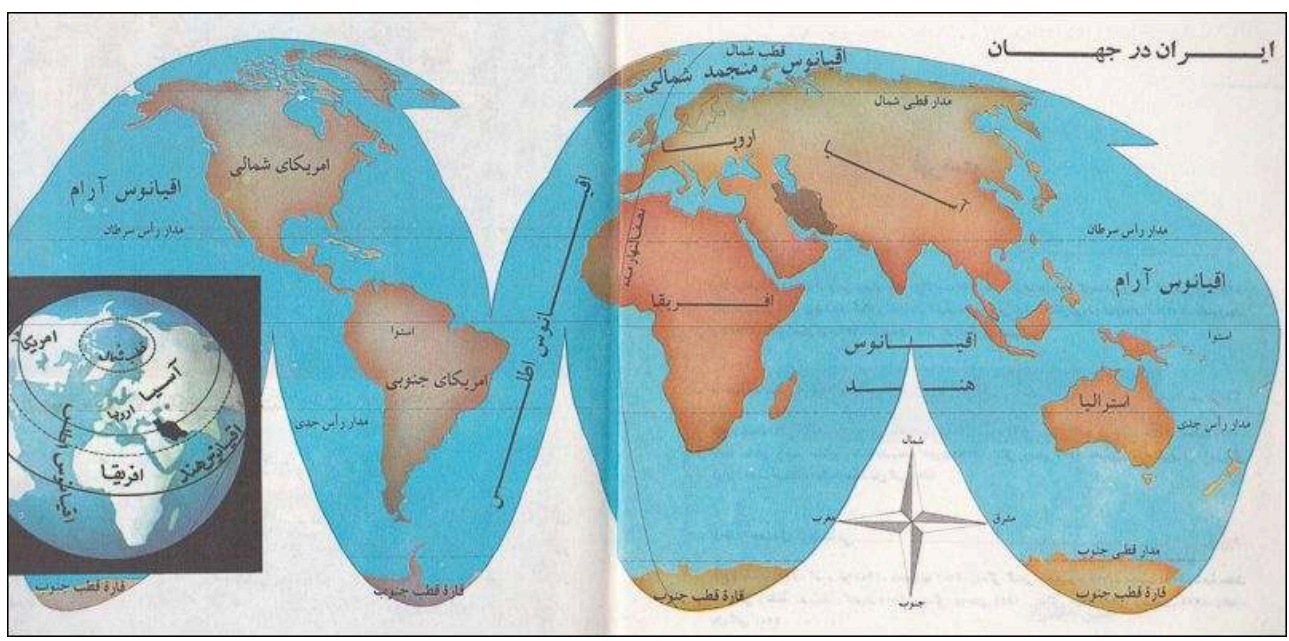

Source : Manuel de géographie, Secondaire, 7 ère année, 1980, p. 2-3.

En 1981, le manuel de géographie de $2^{\mathrm{e}}$ année du secondaire consacré à l'étude du monde débute à nouveau par un planisphère mais le monde n'est pas le principal objet géographique étudié : il est prétexte pour étudier l'Asie. La consigne demande de comparer la superficie de l'Asie à celle des autres continents. Avec ce planisphère, l'accent est mis sur l'effet de rotondité de la terre par l'entremise d'une projection de Robinson (illustration 12). Cette projection a l'avantage de représenter l'ensemble du monde de façon plane tout en donnant l'illusion de la rotondité propre à un globe. Mise au point en 1963, elle est très répandue dans les années 1960 et jusqu'à la fin des années $1980^{15}$.

Illustration 12 - Planisphère selon la projection de Robinson en 1981. Leçon « Asie »

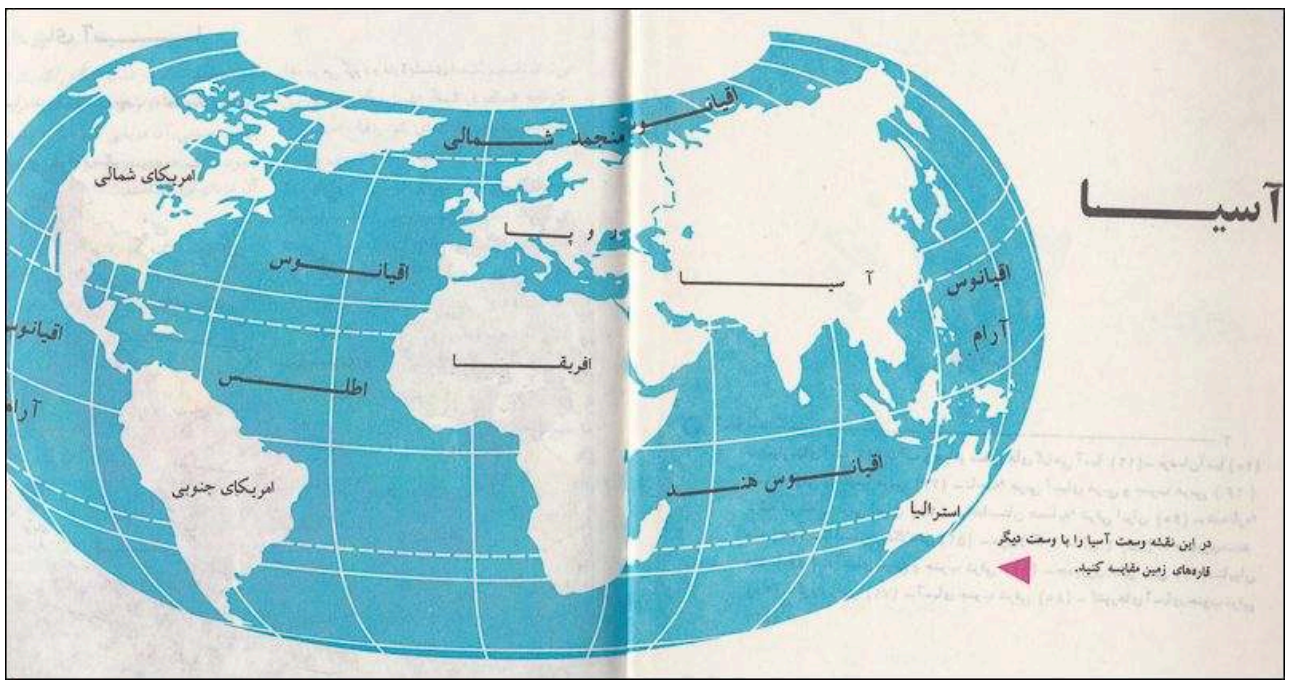

Source : Manuel de géographie, Secondaire, $2^{\text {e }}$ année, 1981, p. 2-3.

Quant au planisphère publié en 2012, il répond à une projection de Gall qui évite les distorsions de surface et conserve les angles des parallèles et des méridiens (illustration 13). Il est quadrillé par les lignes de l'Équateur et du méridien du Greenwich qui accentuent le centrage du planisphère sur un point proche de l'Afrique. 
La projection de Gall est une projection dont certains principes étaient déjà utilisés dans la conception des astrolabes arabes à l'époque médiévale.

Illustration 13 - Planisphère selon la projection de Gall en 2012. Leçon 1 «Comment les océans et les continents sont-ils répartis sur la Terre? »

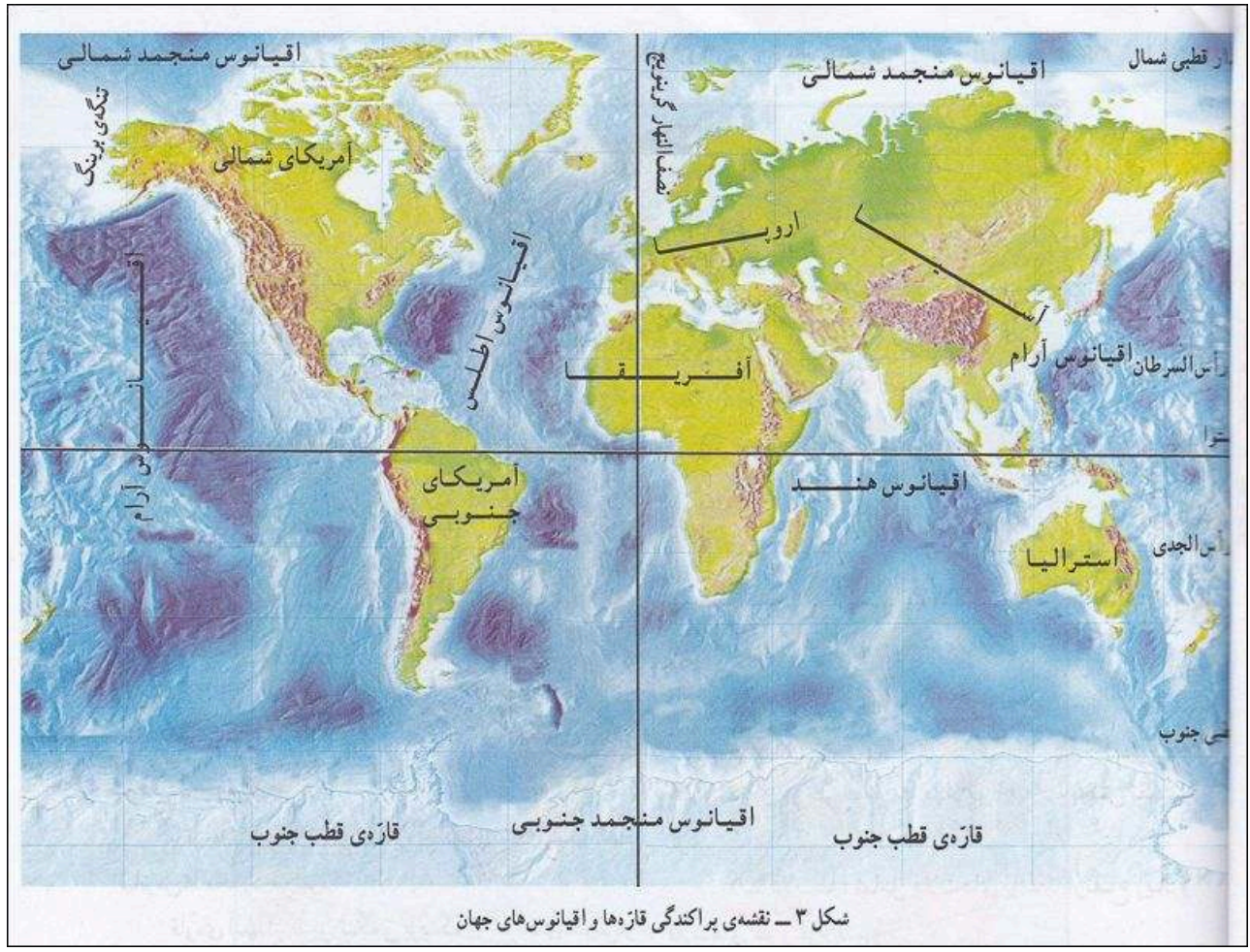

Source : Manuel de géographie, Secondaire, $2^{e}$ année, 2012, p. 3.

Parallèlement à la diffusion du concept de mondialisation, la cartographie pédagogique connaît dans les années 1980 à l'échelle mondiale un renouvellement de ses projections. Contrairement aux manuels publiés dans les pays arabes voisins ou plus loin dans le monde latino-américain - qui continuent un certain temps à adopter un système de représentation classique -, l'Iran participe d'emblée à ce mouvement de refonte cartographique. Ce faisant, en évitant la projection de Mercator et les centrages sur l'Europe, la géographie scolaire iranienne affiche son indépendance par rapport aux cartes, outils et projections de mise en Occident ou au sein de son aire d'influence. Mais, si le choix des projections est novateur, les cadrages restent classiques: les planisphères sont tous orientés au Nord et mettent l'Amérique à gauche.

\section{L'Iran en Asie}

Dans le manuel de 1980, l'Iran se positionne par rapport à l'Asie (Asia), entendue comme la partie du continent eurasiatique distincte de l'Europe. A la fin du chapitre introductif, une page récapitulative de «l'essentiel » à retenir - la superficie, la population et la production de pétrole - est référée à l'Asie au moyen d'une carte du continent et d'un tableau-schéma comparatif qui joue sur l'emboîtement pays/ continent par le dessin proportionnel de figurés et pictogrammes. 
Illustration 14 - Leçon « Iran en Asie »

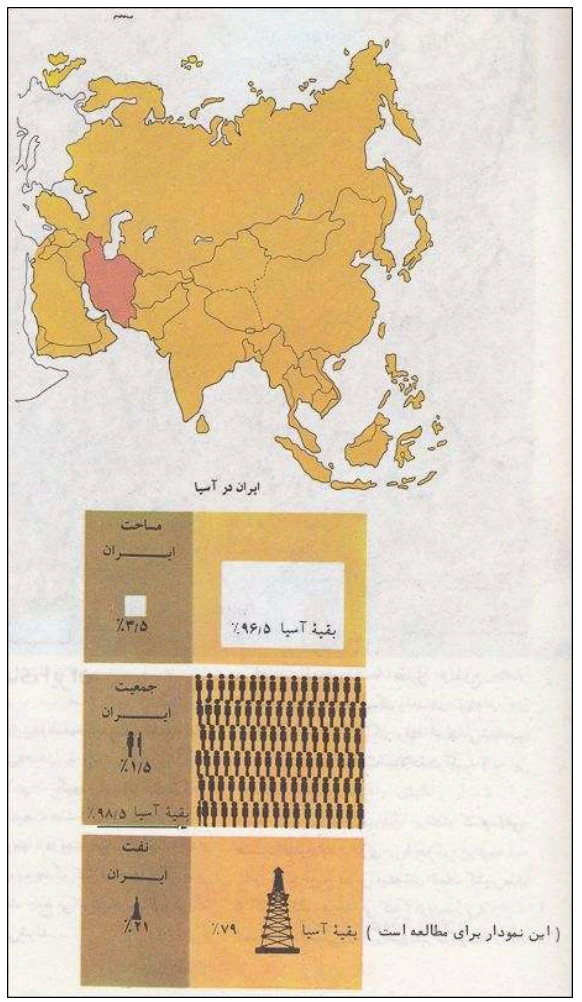

Source : Manuel de géographie, Secondaire, 7 ère année, 1980, p. 13.

Ce sentiment d'appartenance asiatique fait lui-même l'objet d'un niveau d'échelle inférieur (illustration 15). Le manuel scolaire emploie l'étiquette "Asie du Sud-Ouest » (Asia-ye djonub-e gharb) et marque cet ensemble macro-régional d'un rectangle sur la carte. Celui-ci regroupe douze pays : l'Iran, le Proche-Orient, la Turquie et les pays du Centre et du Nord de la péninsule arabique (sans l'Egypte, le Yemen et Oman). Après le rappel des différences démographiques, les liens linguistiques et religieux sont avancés pour justifier une « union » (peyvastegi) des pays de la zone. Mais, au-delà des facteurs culturels, c'est surtout le pétrole qui est prôné comme ferment commun par le biais d'un pictogramme non légendé symbolisant une installation pétrolière de surface. À la fin du texte, l'« Asie du Sud-Ouest » est différenciée de l'expression " Moyen-Orient » (khavarmianeh) considérée comme forgée de toute pièce par les Européens. Or, le qualificatif "Asie du Sud-Ouest» est aussi issu d'un découpage inventé par les Européens mais les auteurs du manuel n'en sont visiblement pas conscients. 
Illustration 15 - Leçon " L'Iran et les pays d'Asie du Sud-Ouest »

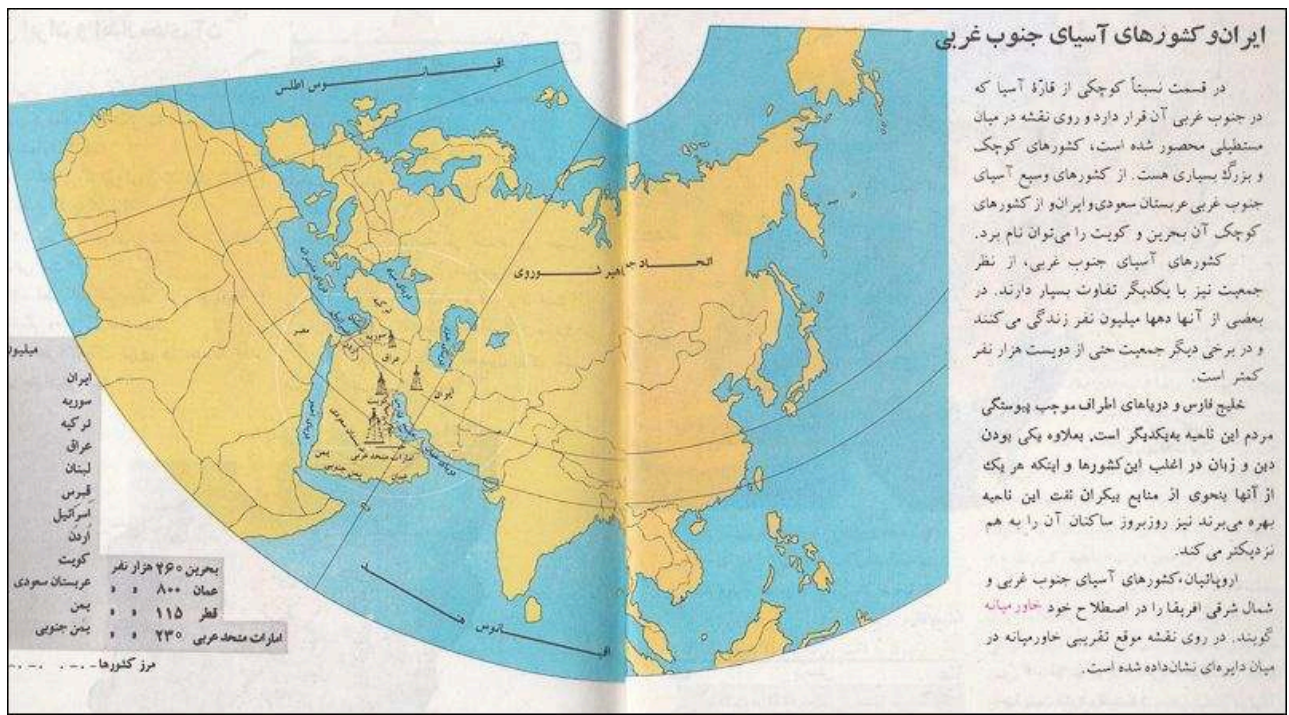

L'Iran et les pays d'Asie du Sud-Ouest (Iran va keshvarha-ye Asia-ye djonub-e gharbı)

Dans la partie relativement petite du continent asiatique qui se trouve au Sud-Ouest et qui sur la carte a été entourée par un rectangle [blanc], il y a des pays petits ou grands. Parmi les pays les plus vastes d'Asie du Sud-Ouest, il y a l'Arabie Saoudite et l'Iran et parmi les pays les plus petits, il y a le Bahrein ou le Koweit.

Du point de vue de la population, les pays d'Asie du Sud-Ouest présentent aussi beaucoup de différences. Dans certains vivent des dizaines de millions de personnes, dans d'autres la population se résume à moins de 200000 individus.

Dans la région du Golfe persique et des mers adjacentes, la source de l'union des personnes est due à I'une ou l'autre de ces causes: outre la religion et la langue qui est commune à la plupart de ces pays, il y a le fait que chacun d'une manière ou d'une autre tire profit du pétrole au point que les habitants de cette région sont de jour en jour plus proches.

Les Européens utilisent l'expression « Moyen-Orient » (khavarmianeh) pour qualifier les pays d'Asie du Sud-Ouest et de l'Afrique du Nord-Est.

Source : Manuel de géographie, Secondaire, 7 ère année, 1980, p. 10-11.

La géographie scolaire iranienne tente de se différencier des projections et des vocables employés en Occident. Elle privilégie un découpage macro-régional qui lui est propre : l'appartenance à l'«Asie du Sud-Ouest». Symbolisant une communauté de pays pétroliers, ce découpage répond à des critères géopolitiques et stratégiques : il exclue notamment le sultanat d'Oman, pro-occidental et dont la population est majoritairement ibadite (ni sunnite ni shiite).

\section{Quatre périphéries-continents}

Les ensembles géographiques considérés comme étrangers au centre iranien et asiatique sont abordés par continent avec plus ou moins de distanciation. Une constante de 1980 à 2012 est l'étude de l'Afrique et de l'Océanie. En 2012, après avoir été éclipsées dans les années 1980, l'Europe et l'Amérique sont étudiées à nouveau; l'Antarctique est introduit, faisant le pendant aux programmes français qui abordent depuis 2010 au lycée « Les mondes arctiques, une nouvelle frontière sur la planète ». 
Nombre de pages réservé à chaque continent dans les manuels de géographie de $2^{\text {ème }}$ année du secondaire (1981 et 2012)

\begin{tabular}{|l|l|l|l|l|l|}
\hline $\begin{array}{l}\text { Périphéries- } \\
\text { continents }\end{array}$ & $\begin{array}{l}\text { Nombre de pages } \\
\text { dévolu dans le } \\
\text { manuel de 1981 }\end{array}$ & $\begin{array}{l}\text { Pourcentage du } \\
\text { nombre de page } \\
\text { dévolu en 1981 }\end{array}$ & $\begin{array}{l}\text { Nombre de pages } \\
\text { dévolu dan le le } \\
\text { manuel de 2012 }\end{array}$ & $\begin{array}{l}\text { Pourcentage du } \\
\text { nombre de page } \\
\text { dévolu en 2012 }\end{array}$ \\
\hline Asie & 84 & $72 \%$ & 18 & $26 \%$ \\
\hline Afrique & 26 & $22 \%$ & 11 & $16 \%$ \\
\hline Europe & 0 & $0 \%$ & 14 & $20,5 \%$ \\
\hline Amérique & 0 & $0 \%$ & 11 & $16 \%$ \\
\hline Océanie & 6 & $5 \%$ & 6 & $9 \%$ \\
\hline Antarctique & 0 & $0 \%$ & 5 & $7 \%$ \\
\hline
\end{tabular}

L'étude des périphéries-continent est moins approfondie que celle du centre asiatique, même si un ré-équilibrage est constatable dans le manuel de 2012. L'étude de l'Afrique occupe une place privilégiée, derrière l'Asie, mais cède le pas quantitativement à l'Europe en 2012.

C'est à l'Afrique qu'est dévolue la place de première périphérie-continent. Ce continent a entretenu des liens historiques avec l'Iran, où de nombreux esclaves ont été déportés au Moyen Âge avant l'instauration de la Traite des Noirs par les Occidentaux au $\mathrm{XVI}^{\mathrm{e}}$ siècle. Le Sud de l'Iran compte aujourd'hui une minorité descendant de ces esclaves. En 1981, le relief, le climat, la densité démographique et la division des États d'Afrique sont tout d'abord étudiés à l'échelle du continent puis une étude régionale est menée mettant l'accent sur l'Afrique du Nord, la vallée du Nil et la production pétrolière en Algérie. Les quelques photographies inclues dans le chapitre de ce manuel réservé à ce continent représentent des archétypes de l'identité africaine : beaucoup de paysages (les chutes Victoria sur le fleuve Zambèze, une vue des dunes dans le Sahara), mais aussi un pygmée et ses pièges traditionnels de chasseur ou un enfant noir qui sourit, loin d'une image misérabiliste. La légende de cette dernière illustration est à connotation raciale: "sur 10 Africains, 6 ont la peau noire ${ }^{16}$. L'étude régionale disparaît dans le manuel de 2012. L'Afrique est abordée en tant qu'entité continentale, du point de vue de son relief, de son climat, de sa population et de son économie. Les ressources et activités économiques sont résumées par une carte dite "illustrée " (naqsheh-ye mosavvar) dans le manuel, c'est-à-dire en symboles, qui fait aussi office de carte de synthèse en fin de leçon (illustration 16). Malgré les pictogrammes éloquents, le résultat est difficilement lisible et ne donne à voir que des tendances. Cette carte fait la part belle aux représentations populaires et aux stéréotypes en caractérisant grossièrement. Il est à noter que les toponymes des pays sont écrits systématiquement en persan et ne suivent pas la sémiologie graphique occidentale, pourtant répandue pour les cartes de l'Afrique. Néanmoins, le dessin des pictogrammes est occidentalisé, répondant à des critères réalistes, comme le dessin du diamant ou de l'arbre. 
Illustration 16 - Carte illustrée de l'économie africaine (naqsheh-ye mosavvar-e eqtesadi-e Afriqa). Leçon 12 « Pourquoi le continent africain est-il exportateur de matières premières et agricoles? "

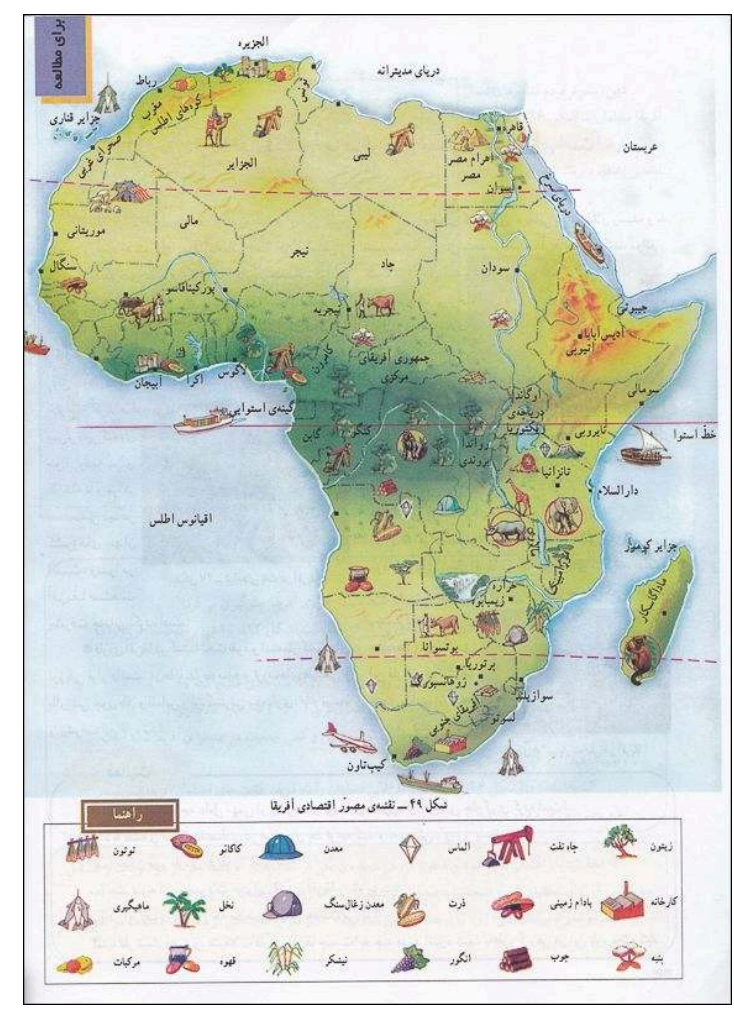

Source : Manuel de géographie, Secondaire, $2^{\mathrm{e}}$ année, 2012, p. 32.

donné le positionnement anti-occidental du régime islamique, l'étude première de l'Afrique est l'occasion de moins attirer l'attention sur les puissances européenne et américaine. A l'opposé, sous le Shah, l'Europe et l'Amérique étaient mises en évidence dans les salles de classe. En 1974, dans le film Le passager (Mosafer) d'Abbas Kiarostami, ce sont surtout les cartes de ces deux continents qui sont affichées dans la classe d'une école pour garçons ${ }^{17}$. Dans les manuels étudiés, des critiques de ces deux périphériescontinent transparaissent en filigrane. En 2012, l'Europe est considérée d'emblée par la négative comme le «continent le moins étendu du monde » ou comme le continent ayant une légitimité douteuse puisqu'il fait partie au sens strict du continent asiatique. Elle est surtout caractérisée par son climat, sa végétation, la couleur verte. La botte de l'Italie fait partie d'un exercice de reconnaissance du tracé des frontières. La population européenne est abordée seulement par une carte de la densité et la photographie de l'Église du Vatican mais aussi d'une mosquée dans les Balkans. La dernière leçon consacrée à l'économie s'avère la plus fournie en illustrations: tracteur dans une exploitation agricole intensive, TGV, industrie de papier en Suède, Big Ben à Londres, station de sport d'hiver et littoral industriel en Italie.

Après un bref rappel historique sur Christophe Colomb, l'Amérique du Nord et du Sud sont abordées ensemble. Le continent américain est présenté avant tout sous l'angle du climat et de la végétation, comme un jardin d'Eden, en témoigne un encadré sur ses fruits et légumes exotiques. Exceptés les fruits, les hauts-lieux présentés en illustration sont tous d'ordre paysager: le canal de Panama, une vue satellite du fleuve Amazone, un volcan en éruption non légendé (sans doute le Popocatepelt au Mexique), les séquoias de Californie, la forêt enneigée du Québec. Un seul document a trait à la 
géographie humaine. Il s'agit d'une carte en points des populations de «couleur noire ", de " couleur blanche " et "métissées » à l'échelle continentale. De même que pour l'Afrique, le critère racial plutôt que culturel revêt une importance première quand il s'agit de donner à imaginer les populations qui vivent dans ces territoires lointains. La dernière leçon interroge à nouveau l'avancée économique. Ici seulement, il est fait spécifiquement allusion à l'Amérique du Nord. Pourtant, alors que l'espace Nord-américain est annoncé dans le titre de la leçon ("Quels sont les facteurs à l'origine du progrès économique de l'Amérique du Nord?»), une photographie de paysans cubains récoltant à la main la canne à sucre est montrée à côté de celle d'un centre commercial de Vancouver au Canada. S'agirait-il de démentir la réalité de ces progrès économiques ou les États-Unis sont-ils encore plus ou moins tabous en 2012 ? Plusieurs auteurs ont montré qu'un esprit de djihad anti-occidental a pu être diffusé à travers les pages de manuels scolaires iraniens, les termes " ennemis », « infidèles » ou « agresseurs » étant parfois utilisés pour qualifier les relations de l'Iran avec l'Occident (Groiss and Toobian, 2006, p. 16-18 ; Carré, 1981, p. 107-108). Dans le manuel de 2012, le discours tenu sur l'Europe et l'Amérique n'est pas ouvertement anti-occidental mais nous pouvons remarquer deux types de dichotomie qui renforcent la distanciation : le contraste pays développé/pays en voie de développement et l'opposition monde musulman/monde chrétien, ou non tout à fait chrétien étant donné la photographie de la mosquée des Balkans pour caractériser l'Europe.

Quant à la périphérie des confins du monde, l'Océanie, elle est introduite dans les manuels de 1981 et 2012 par une carte de la Terre en projection azimutale oblique. Celle-ci est centrée en 1981 sur la Nouvelle-Zélande tandis qu'en 2012, l'accent est mis sur l'Antarctique (non étudié en 1981). Ce dernier continent illustré par des cartes en projection polaire prend de l'importance: une périphérie amenée à devenir un centre? De même qu'en France, sa perception est ambiguë, entre protection et exploitation.

\section{Conclusion}

Cette étude de huit manuels scolaires publiés en Iran entre 1973 et 2012 a montré la place essentielle qu'occupe la carte dans la géographie scolaire iranienne malgré les changements de régimes politiques. Dans ces manuels, la carte ne correspond pas à la définition de mise dans la géographie française, en tant que système d'expression mixte, agençant des éléments d'information en langage verbal et graphique, et entretenant un lien d'analogie avec l'espace. Dans le contexte iranien, la carte est insérée dans un système d'expression auto-référé, étant générique ou muette, exempte de titre, légende ou échelle. Elle répond à une vision de l'espace et du monde qui est intrinsèquement différente de la nôtre - même si la géographie scolaire est le fruit en Iran d'une greffe du système scolaire occidental. Si nous devions analyser ces cartes selon nos référents français, nous déduirions que celles-ci sont affiliées à la géographie post-vidalienne sans pour autant s'inscrire dans le courant de l'analyse spatiale qui a innervé les pratiques pédagogiques en France dans les années 1970, favorisant l'abstraction et une approche problématisée de l'expression cartographique. Mais si ces cartes peuvent paraître désuètes par leur dimension descriptive et leurs techniques graphiques, elles sont toutefois originales dans leurs centrages, variations de projection et découpages. 
38 En Iran, le contenu du savoir véhiculé est principalement en lien avec les intérêts nationaux et connoté par l'idéologie politico-religieuse du régime en place. Le rôle joué par la carte - dont l'usage est si insistant - est double : outil rationnel d'investigation et accès imagé à un univers discursif. D'une part, c'est une banque d'informations localisées qui répond à la question « où? ». D’autre part, par divers recours l'« imprégnation », l'escamotage de certains lieux, la mise en exergue de thématiques, l'ambiguité des découpages, l'assignation de paramètres positifs ou négatifs aux espaces étudiés -, la carte ne se contente pas de faciliter la lecture du manuel en fournissant des images mentales, elle infléchit la compréhension de l'espace en donnant accès à une réalité particulière conçue par son auteur. Ces scénarios cartographiques dénotent la volonté de l'État et des organismes scolaires ${ }^{18}$ de créer une identité territoriale forte pour fédérer une société pluriethnique : ils contribuent à l'iranisation de la société.

39 En France, en Iran, comme ailleurs, idéologie et stratégie influencent discours scolaire et expression cartographique, la carte étant utilisée pour convaincre d'une vision du monde ou de la justesse du discours qui en découle. Il y a plus d'un siècle en Terminale (programme du 31 mai 1902), nous étudions en France les «Grandes Puissances du Monde ». Les programmes d'histoire-géographie des années 2000 ont introduit de nouveaux objets géographiques mais il est encore question des «chemins de la puissance ». De même, la vision de l'espace géographique en Iran s'inscrit dans un système qui instrumentalise la finalité civique de la géographie scolaire. L'importance octroyée au tracé des frontières, aux ressources pétrolières et à un découpage régional favorisant la condensation de certains hauts-lieux identitaires révèle combien les enjeux politiques imprègnent la discipline pour « faire nation ». La désignation en zone concentrique d'un espace d'appartenance relié à l'Asie (l'Iran se conçoit avant tout « asiatique » quand nous le percevons « oriental ») en recourant le moins possible aux notions et projections occidentales permet de recréer une aire d'influence de l'iranité sur les vestiges des anciens empires perses. Quant aux autres continents, ce sont des périphéries caractérisées du point de vue physique ou économique mais non du point de vue culturel et humain : il s'agit de sauvegarder le carcan de la société islamique idéale. Il demeure que le point de vue idéologique est plus estompé dans les manuels de 2012. La sémiologie graphique de certaines cartes à cette époque emprunte davantage aux codes de représentation occidentaux avec l'utilisation courante des technologies informatiques mais aussi le recours à des pictogrammes "modernisés ", inspirés des comics américains. Une enquête de terrain dans les salles de classe en Iran serait un bon complément à cette étude des manuels scolaires pour connaître quel sens l'élève donne véritablement à ces cartes.

\section{BIBLIOGRAPHIE}

Ashraf A., 1998. Education VII. General Survey of Modern Education. In Yarshater E., Encyclopaedia Iranica. Costa Mesa, Mazda Publishers, p. 189-196. 
Anquetil G., 1978. Iran : la Révolution est aussi culturelle. Les Nouvelles Littéraires, 56, 7/14, p. 19-26.

Bédouret D., 2012. Les espaces ruraux d'Afrique noire à travers la géographie scolaire: des représentations à l'espace symbolique. Thèse de doctorat, Université de Toulouse-Le Mirail, $524 \mathrm{p}$.

Birashk A., 1998a. Education X. Middle and Secondary Schools. In Yarshater E., Encyclopaedia Iranica. Costa Mesa, Mazda Publishers, p. 202-205.

Birashk A., 1998b. Education XVI. School Textbooks. In Yarshater E., Encyclopaedia Iranica. Costa Mesa, Mazda Publishers, p. 214-216.

Bombardier A., 2013. Iran : de la Révolution culturelle à la « contestation culturelle ». In Boussois S., Moyen-Orient 2012. Bilan géopolitique. Paris, Institut Medea/Éditions du Cygne, p. 24-30.

Bord J.-P., 1998. Le monde arabe : des espaces géographiques aux représentations cartographiques. Thèse de Doctorat, Université de Tours, 705 p.

Brunet R., 1987. La carte, mode d'emploi. Paris, Fayard/Reclus, 269 p.

Carré O., 1981. Notes sur la notion de Djihad dans les manuels scolaires d'Egypte, d'Iran et d'Irak. Etudes Théologiques et Religieuses, 56, 1, p. 107-108.

Copeaux E., 1994. "De l'Adriatique à la mer de Chine » : les représentations turques du monde turc à travers les manuels d'histoire (1931-1993). Thèse de doctorat, Université de Paris 8, 720 p.

Copeaux E., 2000. Une vision turque du monde à travers les cartes de 1931 à nos jours. Paris, CNRS Editions, $240 \mathrm{p}$.

Dustkhah J., Yaghma'I E., 1998. Education III. The traditional elementary school. In Yarshater E., Encyclopaedia Iranica. Costa Mesa, Mazda Publishers, p. 180-182.

Fontanabona J. et al., 2002. Production de croquis en classe de géographie et pratiques innovantes. L'information géographique, juin, p. 167-185.

Ganji M. H., 1988. Jeografia dar Iran. Az Dar ol-Fonun ta Enqelab-e eslami [Géographie en Iran. De Dar olFonun à la Révolution islamique]. Tehran, Astan Quds Razavi Islamic Research Foundation, 706 p.

Grataloup C., 2011. Représenter le monde. La Documentation photographique, 8084, p. 3-63.

Groiss A., Toobian N., 2006. The Attitude to "the Other" and to Peace in Iranian School Books and Teacher's Guides. Mevaseret-Zion, CMIP, 308 p.

Groiss A., 2004. La démocratie en danger : l'enseignement scolaire saoudien. Extraits de manuels scolaires traduits et présentés par Arnon Groiss. Paris, Berg international (titre original : The West, Christians and Jews in Saudi Arabian Schoolbooks), $157 \mathrm{p}$.

Hellot-Bellier F., 2012. L'enseignement, enjeu des missions chrétiennes en Iran (XIX ${ }^{\mathrm{e}}$-début du $\mathrm{XX}^{\mathrm{e}} \mathrm{s}$.). In Verdeil C., Missions chrétiennes en terre d'islam (XVII-XX' s.). Anthologie de textes missionnaires. Turnhout, Brepols, p. 127-162.

Hourcade B., Mazurek H., Taleghani M., Papoli-Yazdi M.-H., 1998. Atlas d'Iran. Montpellier/Paris, GIP RECLUS/La Documentation Française, 235 p.

Hourcade B., 2002. Iran. Nouvelles identités d'une république. Paris, Belin, 223 p.

Leininger C., 2016. L'usage du cas et de l'exemple dans l'enseignement supérieur : pratiques, apprentissages et rapport aux savoirs. Recherches en éducation, 27, 202 p.

Levy J., 2004. La Carte, enjeu contemporain. La Documentation photographique, 8036, p. 1-64. 
Mehran G., 1989. Socialization and Schoolchildren in the Islamic Republic of Iran. Iranian Studies, 22,1, p. 35-50.

Mehran G., 1990. Ideology and Education in the Islamic Republic of Iran. Compare, 20, 1, p. 53-65.

Mehran G., 1992. Cultural Revolution and Educational Transformation in the Islamic Republic of Iran. World Education News and Reviews, 6, 1, p. 9-16.

Mehran G., 1998. Education XXIV. Education in Postrevolutionary Persia : 1979-1995. In Yarshater E., Encyclopaedia Iranica. Costa Mesa, Mazda Publishers, p. 230-233.

Menashri D., 1992. Education and the Making of Modern Iran. Ithaca, Cornell University Press, 352 p. Mohammadi I., 2004. Le rôle de l'école dans la recomposition de l'identité des jeunes Kurdes dans la République islamique d'Iran. Thèse de Doctorat, EHESS, 389 p.

Mottet G., Chevalier J.-P., Picrel M., 1997. Images et construction de l'espace : apprendre la carte à l'école. Paris, Institut National de Recherche Pédagogique, 255 p.

Nahid S., 1983-1984. L'analyse comparative des manuels scolaires français et iraniens du cycle primaire. Éducation comparée, 33-34, p. 101-114.

Niclot D., 2003. Les manuels scolaires de géographie de l'enseignement secondaire : en comprendre les logiques pour mieux les utiliser. Reims, SCEREN-CRDP, $226 \mathrm{p}$.

Paivandi S., 1998. L'individu dans les manuels scolaires en Iran. CEMOTI, 26, p. 219-234.

Paivandi S., 2003. L'évolution du système éducatif iranien : le conflit ouvert entre famille et école. In M. Cosio, Marcoux R., Pilon M., Quesnel A., Education, Famille et Dynamiques démographiques. Paris, CICRED-INED, p. 55-76.

Paivandi S., 2006. Religion et éducation en Iran. L'échec de l'islamisation de l'école en Iran. Paris, L'Harmattan, 226 p.

Paivandi S., 2008a. La France et la naissance de l'école moderne en Iran. In Delfani M., L'Iran et la France malgré les apparences. Paris, Editions Europerse, p. 77-108.

Paivandi S., 2008b. Discrimination and Intolerance in Iran's Textbooks. Washington, Freedom House, $80 \mathrm{p}$.

Paivandi S., 2014. Islamiser l'école. Vacarme, 68, p. 171-182.

Tixier du Mesnil E., 2010. Panorama de la géographie arabe médiévale. In Bresc H., Tixier du Mesnil E., Géographes et voyageurs au Moyen Âge. Paris, Presses universitaires de Paris Ouest, p. 15-27.

Yavari-d'Hellencourt N., 1988. Ethnies et ethnicité dans les manuels scolaires iraniens. In Digard J.-P., Le fait ethnique en Iran et en Afghanistan. Paris, Editions du CNRS, p. 247-265.

Yavari-d'Hellencourt N., 1992. La représentation du « Turc » dans les manuels scolaires iraniens. CEMOTI, 14, p. 53-62.

Wolfs J.-L. et al., 2008. Les différentes conceptions des rapports entre sciences et religions/laïcité, en particulier dans le champ éducatif. Education comparée, 1, p. 15-32.

\section{ANNEXES}

Corpus de manuels iraniens étudié 
Tableau récapitulatif:

\begin{tabular}{|c|c|c|c|c|}
\hline \multicolumn{2}{|l|}{ PRIMAIRE } & \multicolumn{3}{|l|}{ SECONDAIRE ( $1^{\mathrm{er}}$ cycle) } \\
\hline $1^{\text {ère }}$ année & $4^{\mathrm{e}}$ année & $1^{\text {ère }}$ année & $2^{\mathrm{e}}$ année & $3^{\mathrm{e}}$ année \\
\hline Manuel de persan, 1976 & Manuel de persan, 1973 & & & \\
\hline & & & Manuel de géographie, $\mathrm{n}^{\circ} 1,2012$ & Manuel de géographie, $\mathrm{n}^{\circ}$ \\
\hline
\end{tabular}

Sources :

Manuel du primaire (dabestan) de persan (farsi) - Première année (sal-e avval)

Auteur : Inconnu

Publication : Ministère de l'Éducation et de l'Enseignement de la Monarchie Pahlavi

Homayouni

Edition : Sazman-e ketabha-ye darsi-e iran (Établissement des livres scolaires d'Iran)

Année : 1355 ou 2535 = 1976-77

Lieu : Non mentionné

Plat arrière couverture : texte au nom du Shah.

Manuel du primaire (dabestan) de persan (farsi) - Première année (sal-e avval)

Auteur : Inconnu

Publication : Ministère de l'Éducation et de l'Enseignement de la République islamique d'Iran

Edition : Inconnue

Année : 1358 = 1979-80

Lieu : Non mentionné.

Manuel du primaire (dabestan) de persan (farsi) - Quatrième année (sal-e tshaharom)

Auteur : Inconnu

Publication : Ministère de l'Éducation et de l'Enseignement de la Monarchie Pahlavi

Homayouni

Edition : Sazman-e ketabha-ye darsi-e iran (Établissement des livres scolaires d'Iran)

Année : 1352 = 1973-74

Lieu : Non mentionné

Plat arrière couverture : texte au nom du Shah.

Manuel du primaire (dabestan) de persan (farsi) - Quatrième année (sal-e tschaharom)

Auteur : Inconnu

Publication : Ministère de l'Éducation et de l'Enseignement de la République islamique d'Iran (mais sans logo encore)

Edition : Inconnu

Année : 1358 = 1979-80

Lieu : non mentionné

Manuel du secondaire (rahnama'i) de géographie (djeografi) - Première année (sal-e avval) -

«Études générales secondaires »

Numéro plat avant couverture : 107 
Auteur : Hosein Khalilifar

Publication : Ministère de l'Éducation et de l'Enseignement de la République islamique d'Iran

Edition : Maison d'édition Sepehr (Ciel)

Année $: 1359$ = 1980-81

Lieu : Non mentionné

Plat AR couverture : «Valeur (baha) dans tout le pays 60 rials »

Manuel du secondaire (rahnama'i) de géographie (djeografi) - Deuxième année (sal-e dovvom) - "Études générales secondaires"

Numéro plat avant de couverture : 161

Auteur : Hosein Khalilifar

Mise en page : Parvin Sadr

Publication : Ministère de l'Éducation et de l'Enseignement de la République islamique d'Iran

Edition : Sherkat-e tschap o nashr-e Iran (Société d'impression et d'édition d'Iran)

Année : 1360 = 1981-82

Lieu : Non mentionné

Plat arrière de couverture : "Prix (qeymat) dans tout le pays 53 rials »

Manuel du secondaire (rahnama'i) de géographie (djeografi) - Deuxième année (sal-e dovvom) - "Études générales secondaires"

Numéro plat avant de couverture : 161

Auteurs : Dr. Siavash Sayan, Dr Mehdi Tschubineh, Mansur Malek 'Abbas, Dr Nahid Felahian et Mas'umeh Azadmehr

Publication : Ministère de l'Éducation et de l'Enseignement de la République islamique d'Iran

Edition : Sherkat-e tshap va nashr-e ketab-e darsi-ye iran (Société d'impression et d'édition des livres scolaires)

Année : 1391=2012-13

Lieu : Téhéran

Plat AR de couverture : texte intitulé « Iran sur la voie de la construction » (photo bateau conteneur). Valeur : 5500 rials.

Manuel du secondaire (rahnama'i) de géographie (djeografi) - Troisième année (sal-e sevvom) - «Études générales secondaires »

Numéro plat avant de couverture : 137

Auteurs : Dr Siavash Sayan, Dr Mehdi Tschubineh, Dr Shukat Moqimi, Mansur Malek

'Abbas, Kurosh Amirinia

Publication : Ministère de l'éducation et de l'Enseignement de la République islamique d'Iran

Edition : Sherkat-e tshap va nashr-e ketab-e darsi-ye iran (Société d'impression et d'édition des livres scolaires)

Année : 1391=2012-13

Lieu : Téhéran

Plat arrière de couverture : texte et carte Iran. Valeur : 7000 rials. 


\section{NOTES}

1. L'objectif est d'offrir un aperçu de ce qui était publié à la fin du régime impérial, au tournant de la Révolution de 1979 et plus de trente ans après l'avènement de la République islamique. Voir Descriptif du corpus de manuels en annexe.

2. Soit le dispositif général de représentation cartographique, incluant les modalités de figuration de l'espace et de présentation du fond de carte, ainsi que les habits de la carte.

3. Le système d'enseignement traditionnel en Iran est constitué d'une part des maktab, "maison de l'écriture ». À partir de 5 ou 6 ans, les enfants sous la tutelle d'un mollah (religieux) ou d'un mo'allem (non religieux) y suivent une éducation élémentaire puis éventuellement intermédiaire. Il existe deux types d'implantation de maktab en fonction de l'origine sociale des élèves : au sein du foyer de familles aisées et au sein des mosquées, takiyeh (espaces réservés au théâtre shiite) ou de la maison des professeurs. La particularité de ces établissements a résidé en leur importante couverture territoriale, même si le nombre total d'élèves scolarisés est resté modeste, connaissant un pic de 60008 élèves répartis dans 3329 maktab en 1935 contre 28942 élèves répartis dans 1301 maktab en 1925 (Dustkhah and Yaqma'i, 1998, p. 182). L'enseignement traditionnel est basé sur l'apprentissage de l'alphabet arabo-persan et de rudiments de mathématiques. La lecture et la récitation du Coran représentent la plus grande part. Les élèves ne sont pas classés par âge ou niveau (Paivandi, 2008a, p. 78-79). D'autre part, il existe un autre type d'établissement, la madreseh, dédié aux études supérieures. Fréquentée par l'élite à partir de 14 ans, la madreseh n'est pas réservée à une tranche d'âge précise. L'enseignement des matières religieuses y est privilégié mais aussi la grammaire, la poésie, la calligraphie, le calcul et le savoirêtre (Paivandi, 2008a, p. 79-80).

4. Cette dissociation de la géographie et de l'histoire diffère du modèle français. Il reste à déterminer si cela a été mis en place dès l'origine ou après 1945 lors des réformes éducatives menées sur le modèle anglo-saxon.

5. Grataloup C., 1998. Sous l'influence des conventions scolaires. Espaces Temps, 66/67, p. 71. Cité par J. Fontanabona et alii, 2002, p. 168.

6. Les cartes analytique, de synthèse ou par anamorphose ne sont pas représentées.

7. L'usage des pictogrammes a été écarté en France à partir du tournant de l'analyse spatiale dans les années 1970 mais l'enseignement de la géographie en France tend comme en Iran à « condenser » le territoire national jusqu'à l'ériger en quasi-personne, ce qui est très différent de la géographie scolaire britannique par exemple.

8. Il reste à déterminer si et selon quels termes les manuels d'histoire - l'histoire scolaire ayant traditionnellement cette fonction importante de fabrique de la nation - expliquent et commentent le tracé des frontières.

9. Selon les dires d'anciens élèves iraniens, le territoire national a pu être symbolisé aussi par la forme d'un chat.

10. Le manuel de géographie de 1980 étudie l'Iran en le découpant aussi en régions naturelles: tout d'abord la région dite "désertique et semi-désertique" (nahieh-ye biabani va nimeh-ye biabani), puis la «région tempérée montagneuse" (nahieh-ye mo'tadel-e kuhestani), enfin la « région tempérée de la Caspienne " (nahieh-ye mo'tadel-e khezeri). Ce découpage en trois régions n'est pas justifié. Il serait à vérifier s'il coïncide avec celui du discours scientifique en Iran. Ces trois régions sont circonscrites sur des cartes par des figurés de surface ou par des effets de cache.

11. Yavari d'Hellencourt, 1988, p. 247-248: sont étudiés des manuels édités en 1984, comme le Livre d'enseignement des sciences sociales (Ta'limat-e Ejtema'i) des trois premières années du primaire et le livre de Géographie de l'Iran de la deuxième année du secondaire, avec son complément de Géographie des provinces. Ce complément incluait 24 livrets traitant séparément la géographie des 
24 régions administratives de l'Iran. Les auteurs de chaque livret sont nommés. Il s'agit de professeurs de géographie de la province concernée.

12. Ce lien tissé entre identité nationale et diversité ethnique s'apparente au traitement de la question des nationalités en URSS puis dans la Fédération de Russie. Cependant, la perspective idéologique demeure quelque peu différente, si ce n'est inversée quant à la présentation de la ville et de la vie urbaine.

13. Mais Téhéran apparaît sur la carte de la vie urbaine de la région désertique.

14. Manuel $1^{\text {ère }}$ année de persan, primaire, 1996, p. 107, in Paivandi, 1998, p. 225.

15. Cette projection a été adoptée par la revue National Geographic durant cette période.

16. Manuel de géographie de $2^{\mathrm{e}}$ année du secondaire, 1981, p. 91.

17. Abbas Kiarostami, Mosafer (« Le passager »), Iran, 1974 : vue de la classe à 6'10".

18. En 1963, le gouvernement impérial a instauré un monopole sur la publication des manuels et créé plusieurs organismes affectés à cette tâche : l'Organisation pour la Recherche sur les Livres scolaires (Sazeman-e ketabha-ye darsi) et la Compagnie d'édition ofset (Sherkat-e tchap-e ofset). Ces organismes ont été développés après la Révolution. A la fin des années 1990, l’Organisation pour la Recherche sur les Livres Scolaires (Sazeman-e katabha-ye darsi), au sein du Ministère de l'Education et de l'Enseignement, rassemblait environ vingt groupes de recherche, chacun chargé de réviser les manuels de toutes les disciplines (Birashk, 1998, p. 215). Certains de ces groupes comptent des ulémas (Merhran, 1989, p. 37-8). Une nouvelle instance a d'ailleurs été créée en 1979, les Affaires éducatives (Omour-e tarbiyati), pour vérifier en particulier si la culture islamique est bien inculquée aux élèves (Paivandi, 2014).

\section{RÉSUMÉS}

À travers un corpus de huit manuels de géographie et persan, publiés en Iran avant ou après la Révolution islamique et supports de l'école primaire ou secondaire, cet article analyse la place de la carte du point de vue graphique, sémiologique et discursif. Des scénarios cartographiques originaux sont mis en lumière. La situation de la carte dans le système d'images mais aussi son rôle dans l'«iconicisation» du territoire national et dans la représentation du monde sont présentés. Cette exploration des procédés de la cartographie scolaire en Iran permet de mettre en exergue la contribution de la géographie à la fabrique du territoire et de l'identité nationale dans une société multi-ethnique et multi-linguistique.

Through a corpus of eight textbooks of Geography and Persian language, published in Iran before or after the Islamic Revolution and reserved for primary or secondary school, this article analyses the rank of the map from a graphic, semeiological and discursive point of view. Original cartographic scenarios are highlighted. Both the situation of the map in the image system and its role in the "iconicisation" of the national territory and in the representation of the world are presented. This examination of school cartography processes in Iran brings out the contribution of geography to the territory and national identity production in a multi-ethnic and multilinguistic society. 
INDEX

Mots-clés : Iran, carte, image, représentation, géographie scolaire, nation

Keywords : Iran, map, image, representation, school geography, nation

Thèmes : Sur le Champ - Sur le Terrain

\section{AUTEUR}

\section{ALICE BOMBARDIER}

Alice Bombardier, alice.bombardier@gmail.com, est chercheure associée au CADIS (EHESS-CNRS) et agrégée de géographie. Elle a publié récemment :

- Bombardier A., 2017. Les pionniers de la Nouvelle peinture en Iran. Euvres méconnues, activités novatrices et scandales au tournant des années 1940. Berne, Peter Lang, 362p.

- Bombardier A., Montua G., 2017. « À l'est de Chypre - La Vénus de Botticelli chez Āghdāshlū, Karamustafa et Osūlī ». Revue des Mondes Musulmans et de la Méditerranée [En ligne], 142. https:// journals.openedition.org/remmm/10071

- Bombardier A., 2017. « Twentieth-Century Mutations of Persian Miniature Painting: A Testimony from the Iranian Miniaturist Hosein Behzad ». In Ritter M., Sturkenboom I., Valdéz Fernandéz F. (ed.), Beiträge zur Islamischen Kunst und Archäologie. Wiesbaden, Reichert Verlag, p. 149-166. 